TRANSACTIONS OF THE

AMERICAN MATHEMATICAL SOCIETY

Volume 359, Number 3, March 2007, Pages 1375-1401

S 0002-9947(06)04220-6

Article electronically published on October 24, 2006

\title{
COMPUTING O-MINIMAL TOPOLOGICAL INVARIANTS USING DIFFERENTIAL TOPOLOGY
}

\author{
YA'ACOV PETERZIL AND SERGEI STARCHENKO
}

\begin{abstract}
We work in an o-minimal expansion of a real closed field. Using piecewise smoothness of definable functions we define the topological degree for definable continuous functions. Using this notion of the degree we obtain a new proof for the existence of torsion points in a definably compact group, and also a new proof of an o-minimal analogue of the Brouwer fixed point theorem.
\end{abstract}

\section{INTRODUCTION}

We work in an o-minimal expansion $\mathcal{R}$ of a real closed field $\mathbf{R}$.

In the last few years there has been a significant progress in applying ideas and methods from algebraic topology to sets definable in $\mathcal{R}$ (see, for example, [1], 2], [1], 6], 20]). Probably one of the most important results in this direction was a positive answer by M. Edmundo to the following problem posted by the first author and C. Steinhorn (see 15]).

The Torsion Point Problem. Let $G$ be a definable group. If $G$ is definably compact, then $G$ has a $p$-torsion point for every prime $p$.

In fact, using methods of algebraic topology, M. Edmundo and M. Otero in [6] gave a precise count of torsion points for abelian definably compact groups. This count of the torsion points required the introduction of classical invariants, such as orientation and degree of a map, into the o-minimal setting. It was done, in the category of definable $C^{0}$-manifolds and continuous maps, using definable homology.

In this paper we propose more tangible definitions of orientation and degree. Our approach is based on piecewise differentiability of definable functions. For example, if $U$ is a definable open subset of $\mathbf{R}^{n}$ and $f: U \rightarrow \mathbf{R}^{n}$ is a definable continuous locally injective map, then, outside of a set of small dimension, $f$ is differentiable and has a nonsingular Jacobian. We prove in Section 3.1 that if, in addition, $U$ is definably connected, then the determinant of the Jacobian of $f$ has a constant sign on $U \backslash X$, for some $X$ of small dimension. It allows us, using the sign of the determinant of the Jacobian of $f$, to decide whether $f$ is orientation preserving or reversing.

In Section 3.2 we introduce a notion of orientability for definable manifolds that is based on comparing the signs of determinants of Jacobians of transition maps.

Received by the editors June 20, 2005.

2000 Mathematics Subject Classification. Primary 03C64, 03C98, 57R99.

Key words and phrases. O-minimality.

The second author was supported in part by NSF Grant \#0400163.

(C)2006 American Mathematical Society Reverts to public domain 28 years from publication 
In Section 3.3 we show that this notion of orientation for definable manifolds coincides with combinatorial orientation based on triangulation. As was proved in [2], in the case of definably compact manifolds, the combinatorial orientation coincides with one obtained via local homology groups. Thus, at least in the definably compact case, all three notions of orientation agree.

In Section 3.4 we define a notion of degree for definably proper maps between definable oriented manifolds of the same dimension, based on analogous definition for differentiable maps. Once again, as in the case of orientation, the definition of degree is homology-free.

In Section 3.5, as an illustration of our approach, we give another proof of Brouwer fixed point theorem in the o-minimal setting.

In Section 4 we turn to definable smooth manifolds. The main goal in this section is to give a homology-free solution to the Torsion Point Problem. We do it by using methods from differential geometry. As in the classical case, we compute the Euler characteristic of a definable smooth manifold using Morse functions. For example, we prove that every definably compact $C^{4}$-manifold with trivial tangent bundle has zero Euler characteristic. It implies that every definably compact group has Euler characteristic zero and therefore (see [19]) a positive answer to the Torsion Point Problem. Using Morse function we also prove that for a definably compact $C^{4}$ manifold, the Euler characteristic equals Lefschetz number. It provides a missing link between the topological point of view and the differential one, which was left as an open question in [1].

The proofs of the properties of Morse functions that we are using are almost identical to the corresponding proofs in the classical case $\mathbf{R}=\mathbb{R}$. We present them in Appendix A.

Part of the work on this paper was done when the second author was visiting McMaster University (Hamilton, Canada), and also during the semester in Model Theory and Applications to Algebra and Analysis at Isaac Newton Institute for Mathematical Sciences (Cambridge, UK). We thank them for their hospitality. We also thank Margarita Otero for her helpful comments on an earlier draft of this paper.

\section{Preliminaries}

We fix an o-minimal expansion $\mathcal{R}$ of a real closed field $\mathbf{R}$. For convenience we will assume that $\mathcal{R}$ is $\omega$-saturated, however, all our results hold in an arbitrary o-minimal expansion of a real closed field.

For definitions and basic properties of o-minimal structures we refer to [4].

We assume familiarity with definable $C^{p}$-manifolds. For a definition of a definable $C^{p}$-manifold we refer to [1, Section 10.1] where they are called abstractdefinable $C^{p}$-manifolds. We also refer to [15, 14 for additional information on definable $C^{p}$-manifolds. By a definable manifold we mean a definable topological (i.e., $C^{0}$ ) manifold. We don't assume that a definable manifold is definably connected, however, we will always assume that its definably connected components have the same dimension.

For a differentiable map $f$ from an open subset of $\mathbf{R}^{n}$ to $\mathbf{R}^{m}$, we will denote by $J(f)_{x}$ the Jacobian matrix of $f$ at $x$, and by $\left|J(f)_{x}\right|$ its determinant. 
For a definable manifold $M$ and a subset $V$ of $M$, we will denote by $\operatorname{cl}(V)$ the topological closure of $V$ in $M$. We will also write $\operatorname{cl}_{M}(V)$ if we want to emphasize that the closure is taken in the ambient manifold $M$.

We will frequently use the following result that was proved for o-minimal expansions of fields in 20], and for arbitrary o-minimal structures in 8 .

Theorem 2.1 (Invariance of Domain). Let $U$ be a definable open subset of $\mathbf{R}^{n}$ and $f: U \rightarrow \mathbf{R}^{n}$ a definable continuous injective map. Then $f(U)$ is open in $\mathbf{R}^{n}$ and $f$ is a homeomorphism between $U$ and $f(U)$.

The following fact follows from [8, Proposition 2].

Fact 2.2. Let $M$ be a definably connected definable manifold of dimension $n$ and $V$ a definable open dense subset of $M$. Consider the graph $\Gamma$ whose vertices are the definably connected components of $V$ with two components $V_{i}$ and $V_{j}$ connected by an edge if and only if $\operatorname{dim}\left(\operatorname{cl}\left(V_{i}\right) \cap \operatorname{cl}\left(V_{j}\right)\right) \geqslant n-1$. Then the graph $\Gamma$ is connected.

2.1. Generic points. We will be using generic points. In this section we review briefly their basic properties and refer to [16, Section 1] for more details.

Let $A \subseteq \mathbf{R}$ and $a \in \mathbf{R}^{n}$. Then $\operatorname{dim}(a / A)$, the dimension of $a$ over $A$, is the least cardinality of a subtuple $a^{\prime}$ of $a$ such that $a \in \operatorname{dcl}\left(A \cup a^{\prime}\right)$.

Let $X \subseteq \mathbf{R}^{n}$ be a set definable over $A \subseteq \mathbf{R}$, and $a \in X$. We say that $a$ is $a$ generic point in $X$ over $A$ if $\operatorname{dim}(a / A)=\operatorname{dim}(X)$.

Let $X_{1}, X_{2}$ be sets definable over $A \subseteq \mathbf{R}$. We say that $a_{1} \in X_{1}$ and $a_{2} \in X_{2}$ are independent generics if $a_{1}$ is generic in $X_{1}$ over $A$ and $a_{2}$ is generic in $X_{2}$ over $A \cup a_{1}$. It is not hard to see that $a_{1} \in X_{1}$ and $a_{2} \in X_{2}$ are independent generics over $A$ if and only if $\left(a_{1}, a_{2}\right)$ is a generic point in $X_{1} \times X_{2}$ over $A$.

Existence of generics. Since we assume that $\mathcal{R}$ is $\omega$-saturated, if $X \subseteq \mathbf{R}^{n}$ is a set definable over finite $A \subseteq \mathbf{R}$, then there is always $a \in X$ generic over $A$.

In any particular proof involving a definable set $X$, we say that $a$ is generic in $X$, if $a$ is a generic point in $X$ over the set of all parameters that have appeared in the proof thus far.

2.2. On local connectedness. For the notion of a definable space and their basic properties we refer to [4, Chapter 10]. The only definable spaces we will need in this paper are definable subsets of definable manifolds. Namely, if $M$ is a definable manifold, then $M$ with its definable atlas is also a definable space; and every definable subset $X$ of $M$ can be considered as a definable subspace of $M$ in the sense of [4, page 158]. Since all such spaces are regular, from now on by definable space we will mean a regular definable space.

In this section we consider the following question:

Let $X$ and $Y$ be definable spaces such that $X \times Y$ is a definable manifold. Does it imply that $X$ is also a definable manifold?

In a private communication, M. Shiota informed us that, for o-minimal structures over the reals, an affirmative answer follows from his $\mathfrak{X}$-Hauptvermutung [18, Chapter 3]. We expect that the answer is also positive for any o-minimal expansion of a real closed field, and in this section we prove it when the dimension of $X$ is 1 .

We first review local connectedness and related notions that, over the real numbers, were introduced in [13. 
For a definable space $X$ we will denote by $\#(X)$ the number of definably connected components of $X$. Since definable connectedness coincides with definable path-connectedness, the following fact is elementary.

Fact 2.3. If $X$ and $Y$ are definable spaces, then $\#(X \times Y)=\#(X) \cdot \#(Y)$.

Let $X$ be a definable space and $x \in X$. For $\alpha>0$, a definable family $\left\{U_{t}, t \in\right.$ $(0, \alpha)\}$ of definable open neighborhoods of $x$ in $X$ is called a good definable basis for the topology of $X$ at $a$ if the following conditions are satisfied:

(1) every $U_{t}$ is definably connected;

(2) $t<t^{\prime}$ implies $U_{t} \subseteq U_{t^{\prime}}$;

(3) for every definable open subset $V$ of $X$ containing $x$ there is $t$ such that $U_{t} \subseteq V$.

Example 2.4. Let $X$ be a definable subset of $\mathbf{R}^{n}$ considered as a definable space and $x \in X$. For $r>0$ let $B(x, r)$ be the open ball of radius $r$ in $\mathbf{R}^{n}$ centered at $x$, and $U_{r}$ the definably connected component of $B(x, r) \cap X$ containing $x$. Then the family $\left\{U_{r}: r \in(0,1)\right\}$, is a good definable basis for the topology of $X$ at $x$.

Since, by [4, Chapter 10 (1.8)], every definable regular space is definably homeomorphic to a definable subspace of $\mathbf{R}^{n}$, the above example shows that in every definable space $X$ each point $x \in X$ has a good definable basis for the topology of $X$ at $x$. Moreover, such a basis can be chosen definably uniformly in $x \in X$.

The proof of the following lemma is contained in [13, Lemma 2.3].

Lemma 2.5. Let $X$ be a definable space, $Y \subseteq X$ a definable subset of $X, x \in X$, and $\left\{U_{t}, t \in(0, \alpha)\right\}$ a good definable basis for the topology of $X$ at $x$. Then for all sufficiently small $t>0$ every definably connected component of $U_{t} \cap Y$ has $x$ in its closure.

Let $X$ be a definable space, $Y$ a definable subset of $X, x \in X$, and $\left\{U_{t}, t \in\right.$ $(0, \alpha)\}$ a good definable basis for the topology of $X$ at $x$. Then, by o-minimality, $\#\left(U_{t} \cap Y\right)=\#\left(U_{t^{\prime}} \cap Y\right)$ for all sufficiently small $t, t^{\prime}$. Moreover, Lemma 2.5implies that if $V_{\tau}$ is another good basis for $X$ at $x$, then $\#\left(V_{t} \cap Y\right)=\#\left(U_{\tau} \cap Y\right)$ for all sufficiently small $t, \tau$. We will denote this number by $\#(Y)_{x}$ (which could be zero if $x \notin \operatorname{cl}(Y))$.

Claim 2.6. Let $X$ be a definable space of dimension one. Then $X$ is a definable manifold if an only if $\#(X \backslash\{x\})_{x}=2$ for all $x \in X$.

Proof. Follows from cell decomposition.

Claim 2.7. Let $M$ be a definable $n$-dimensional manifold and $Y \subseteq M$ a definable subset of dimension $n-1$. Then for every generic $y \in Y$ the number $\#(M \backslash Y)_{y}$ equals 2 .

Proof. Since the number $\#(M \backslash Y)_{y}$ is computed locally in $M$ near $y$, we can work in a definable chart containing $y$ and assume that $M$ is an open subset of $\mathbf{R}^{n}$. Since $y \in Y$ is generic, there is an open $U \subseteq M$ containing $y$ such that $Y \cap U$ is a $C^{1}$ submanifold of $U$. Working locally after applying an appropriate diffeomorphism, we are reduced to the case where $M$ is an open subset of $\mathbf{R}^{n}$ and $Y$ is the intersection of $M$ with the hyperplane $\left\{x_{n}=0\right\}$. Taking open balls centered at $y$ as a good basis for the topology of $U$ at $y$, we obtain \# $(M \backslash Y)_{y}=2$. 
Theorem 2.8. Let $X, Y$ be definable spaces with $\operatorname{dim}(X)=1$ and $M=X \times Y$. If $M$ is a definable manifold, then $X$ is also a definable manifold.

Proof. By Claim 2.6. we need to show that $\#(X \backslash\{x\})_{x}=2$ for any $x \in X$.

We fix $x \in X$ and denote by $Y_{x}$ the set $\{x\} \times Y \subseteq M$. Let $y \in Y$ be generic over $x$. Then $(x, y)$ is generic in $Y_{x}$ and, by Claim 2.7. $\#\left(M \backslash Y_{x}\right)_{(x, y)}=2$. Also, since $y \in Y$, we have $\#(Y)_{y}=1$.

Let $U_{t}$ be a good definable basis for the topology of $X$ at $x$, and let $V_{t}$ be a good definable basis for the topology of $Y$ at $y$. Obviously, $U_{t} \times V_{t}$ is a good definable basis for the topology of $M$ at $(x, y)$. For all sufficiently small $t$ we have

$$
\#\left(\left(U_{t} \times V_{t}\right) \backslash Y_{x}\right)=2 \quad \text { and } \quad \#\left(V_{t}\right)=1 .
$$

Since $\left(U_{t} \times V_{t}\right) \backslash Y_{x}=\left(U_{t} \backslash\{x\}\right) \times V_{t}$, by Fact 2.3 , \# $\left(U_{t} \backslash\{x\}\right)=2$, hence \# $(X \backslash$ $\{x\})_{x}=2$.

\section{ORientation on $C^{0}$-MANifolds, AND the DEgREe of CONTINUOUS MAPS}

3.1. Locally injective maps. Let $f: M \rightarrow N$ be a definable continuous map between definable manifolds, and $a \in M$. We say that $f$ is locally injective at $a$ if there is a definable open neighborhood $U$ of $a$ in $M$ such that $f$ is injective on $U$. We say that $f$ is locally injective if it is locally injective at every $a \in M$.

Let $U \subseteq \mathbf{R}^{m}$ be a definable open set and $f: U \rightarrow \mathbf{R}^{n}$ a definable continuous map. A point $a \in U$ is called a regular point of $f$ if $f$ is $C^{1}$ on a neighborhood of $a$ and the rank of $J(f)_{a}$ equals $n$. We will denote the set of all regular points of $f$ by $\operatorname{Reg}(f)$. Obviously, $\operatorname{Reg}(f)$ is a definable open set. Notice that if $m=n$, then a point $a \in U$ is regular if and only if $f$ is a diffeomorphism from an open neighborhood of $a$ onto an open subset of $\mathbf{R}^{n}$.

Let $U \subseteq \mathbf{R}^{n}$ be a definable open set and $f: U \rightarrow \mathbf{R}^{n}$ a definable continuous locally injective map. It is easy to see that in this case the set $\operatorname{Reg}(f)$ is a dense open subset of $U$ and the function $x \mapsto \operatorname{Sign}\left(\left|J(f)_{x}\right|\right)$ is locally constant on $\operatorname{Reg}(f)$. Our goal is to prove that it is constant on all $\operatorname{Reg}(f)$, provided $U$ is definably connected. (Note that $\operatorname{Reg}(f)$ need not be definably connected itself.)

We will need the following lemma.

Lemma 3.1. Let $W \subseteq \mathbf{R}^{n-1}$ and $U \subseteq \mathbf{R}^{n}$ be definable open sets such that the set $\widetilde{W}=W \times\{0\}$ is contained in the boundary of $U$. Let $F: U \cup \widetilde{W} \rightarrow \mathbf{R}$ be a definable function continuous on $U \cup \widetilde{W}$ and $C^{1}$ on $U$, let $f: W \rightarrow \mathbf{R}$ be the function $y \mapsto F(y, 0)$, and $w \in W$ a generic point. Then $f$ is differentiable at $w$ and, for all $i=1, \ldots, n-1$,

$$
\frac{\partial f}{\partial x_{i}}(w)=\lim _{u \rightarrow(w, 0), u \in U} \frac{\partial F}{\partial x_{i}}(u)
$$

Proof. We will assume $i=1$.

We will denote by $x$ the $n$-tuple of variables $\left(x_{1}, \ldots, x_{n}\right)$ and by $y$ the $(n-1)$ tuple $\left(y_{1}, \ldots, y_{n-1}\right)$.

Since $w$ is generic in $W$, the map $f$ is differentiable near $w$, and we will just assume that $f$ is differentiable everywhere on $W$.

After replacing $F(y, t)$ by $F(y, t)-F(y, 0)$, we may also assume that $f(y) \equiv 0$ on $W$. 
For $x \in U$, let $F^{\prime}(x)$ denote $\frac{\partial F}{\partial x_{1}}(x)$. We need to show that

$$
\lim _{u \rightarrow(w, 0), u \in U} F^{\prime}(u)=0 .
$$

We divide $U$ into two sets $U^{+}=\left\{x \in U: x_{n}>0\right\}$ and $U^{-}=\left\{x \in U: x_{n}<0\right\}$. Obviously, to show (3.1) it is sufficient to prove that for $\epsilon \in\{-,+\}$ if $(w, 0) \in \operatorname{cl}\left(U^{\epsilon}\right)$, then

$$
\lim _{u \rightarrow(w, 0), u \in U^{\epsilon}} F^{\prime}(u)=0 .
$$

We will consider only the case of $U^{+}$and assume $(w, 0) \in \operatorname{cl}\left(U^{+}\right)$.

Since $w$ is generic in $W$, there is an open definable neighborhood $V$ of $w$ in $W$ and $d>0$ such that $V \times(0, d) \subseteq U^{+}$. Shrinking $W$ if needed, we may assume that $W \times(0, d) \subseteq U^{+}$.

Since $(w, 0)$ is a generic point in the boundary of $U^{+}$and $U^{+}$is locally connected at $(w, 0)$, by o-minimality, the limit of $F^{\prime}(u)$, as $u \in U^{+}$approaches $(w, 0)$, exists in $\mathbf{R} \cup\{ \pm \infty\}$. Let $L$ be the limit.

Towards getting a contradiction we assume that $L \neq 0$, say $L>0$. Then, since $w$ is generic in $W$, there is $C>0$ and $\varepsilon_{1}, \varepsilon_{2}>0$ such that the set $\left\{y:\|y-w\| \leqslant \varepsilon_{1}\right\}$ is contained in $W$ and

$$
F^{\prime}(y, t)>C \text { for all }(y, t) \in \mathbf{R}^{n} \text { with }\|y-w\| \leqslant \varepsilon_{1}, 0<t<\varepsilon_{2} .
$$

Since $F$ is continuous on $U \cup \widetilde{W}$ and vanishes on $\widetilde{W}$, for every $y \in W$ there is $\delta(y)>0$ such that $|F(y, t)|<\frac{\varepsilon_{1}}{2} C$ for $0<t<\delta(y)$. The set $\left\{y \in W:\|y-w\| \leqslant \varepsilon_{1}\right\}$ is definably compact, hence we can find $\delta>0$ such that

$$
|F(y, t)|<\frac{\varepsilon_{1}}{2} C \text { on the set }\left\{(y, t):\|y-w\| \leqslant \varepsilon_{1}, 0<t<\delta\right\} .
$$

Decreasing $\delta$ if needed, we will assume $\delta<\varepsilon_{2}$.

Let $w^{\prime}=\left(w_{1}+\varepsilon_{1}, w_{2}, \ldots, w_{n-1}\right), b=(w, \delta / 2)$, and $b^{\prime}=\left(w^{\prime}, \delta / 2\right)$, where $\left(w_{1}, \ldots, w_{n-1}\right)=w$. We have $w^{\prime} \in W$ and $b, b^{\prime} \in U^{+}$. From (3.3) we obtain

$$
\left|F(b)-F\left(b^{\prime}\right)\right| \leqslant|F(b)|+\left|F\left(b^{\prime}\right)\right|<C \varepsilon_{1} .
$$

Consider the map $g(t)=F\left(w_{1}+t \varepsilon_{1}, w_{2}, \ldots, w_{n-1}, \delta / 2\right)$. We have $g(0)=F(b)$ and $g(1)=F\left(b^{\prime}\right)$. By the Mean Value Theorem, there is $\tau \in(0,1)$ such that $g^{\prime}(\tau)=$ $F\left(b^{\prime}\right)-F(b)$, hence $\left|g^{\prime}(\tau)\right|<C \varepsilon_{1}$. Since $g^{\prime}(\tau)=\varepsilon_{1} \frac{\partial F}{\partial x_{1}}\left(w_{1}+\tau \varepsilon_{1}, w_{2}, \ldots, w_{n-1}, \delta / 2\right)$, we obtain

$$
\left|\frac{\partial F}{\partial x_{1}}\left(w_{1}+\tau \varepsilon_{1}, w_{2}, \ldots, w_{n-1}, \delta / 2\right)\right|<C,
$$

contradicting (3.2).

Now we are ready to prove the main theorem of this section.

Theorem 3.2. Let $U \subseteq \mathbf{R}^{n}$ be a definable open definably connected set and $F: U \rightarrow$ $\mathbf{R}^{n}$ a definable continuous locally injective map. Then the function $x \mapsto\left|J(F)_{x}\right|$ has constant sign on $\operatorname{Reg}(F)$.

Proof. Let $V_{1}, \ldots, V_{k}$ be all definably connected components of $\operatorname{Reg}(F)$. Obviously, $\left|J(F)_{x}\right|$ has constant sign on each $V_{i}$. By Fact 2.2, we need to show that for any two components $V_{i}, V_{j}$ with $\operatorname{dim}\left(\operatorname{cl}_{U}\left(V_{i}\right) \cap \operatorname{cl}_{U}\left(V_{j}\right)\right)=n-1$ the sign of $|J(F)|$ on $V_{i}$ is the same as on $V_{j}$. We consider two such components $V_{i}, V_{j}$ and assume they are $V_{1}$ and $V_{2}$. Let $D=\operatorname{cl}_{U}\left(V_{1}\right) \cap \mathrm{cl}_{U}\left(V_{2}\right)$. (Thus $\operatorname{dim}(D)=n-1$.) 
Let $w_{0}$ be a generic point in $D$. By o-minimality, the set $D$ is a $C^{1}$-submanifold of $\mathbf{R}^{n}$ near $w_{0}$. Thus, there is a definable open ball $B \subseteq \mathbf{R}^{n}$ containing $w_{0}$ and a definable diffeomorphism $g$ from $B$ onto an open subset of $\mathbf{R}^{n}$ such that $g(B \cap D) \subseteq\left\{x_{n}=0\right\}$. Shrinking $B$ and composing $g$ with the reflection with respect to the plane $\left\{x_{n}=0\right\}$ if needed, we may assume that $g\left(B \cap V_{1}\right) \subseteq\left\{x_{n}>0\right\}$ and $g\left(B \cap V_{2}\right) \subseteq\left\{x_{n}<0\right\}$.

Since $g$ is a diffeomorphism, the sign of $\left|J(g)_{x}\right|$ is constant on $B$. Hence we may replace $U$ with $g(B \cap U), F$ with $F \circ g^{-1}$ and assume that

$$
V_{1} \subseteq\left\{x_{n}>0\right\}, V_{2} \subseteq\left\{x_{n}<0\right\} \text { and } D \subseteq\left\{x_{n}=0\right\}
$$

Shrinking $U$ again if needed, we may also assume that $F$ is injective on $U$. By the Invariance of Domain Theorem, we have that $F(U)$ is open in $\mathbf{R}^{n}$ and $F$ maps $U$ homeomorphically onto its image.

Let $w_{1}$ be a generic point in $F(D)$. Since $F(D)$ is a $C^{1}$-submanifold of $\mathbf{R}^{n}$ near $w_{1}$, we can apply an appropriate diffeomorphism of an open neighborhood of $w_{1}$ and assume that $F(D) \subseteq\left\{x_{n}=0\right\}$.

As before, we may assume that

$$
F\left(V_{1}\right) \subseteq\left\{x_{n}>0\right\} \text { and } F\left(V_{2}\right) \subseteq\left\{x_{n}<0\right\}
$$

Let $(a, 0)$ be a generic point in $D$. (In particular, $a$ is a generic point in $\mathbf{R}^{n-1}$.) Writing $F=\left(F_{1}, \ldots, F_{n}\right)$, since $F_{n}(a, 0)=0$ and $F_{n}(a, z)$ is increasing in $z$ near 0 , we can find $\varepsilon>0$ small enough such that $\frac{\partial F_{n}}{\partial x_{n}}(a, y)>0$ for all $y \in(-\varepsilon, \varepsilon)$ different from 0 . Since $a$ is generic in $R^{n-1}$, we may assume, by shrinking $U$ if needed, that for all $\left(x_{1}, \ldots, x_{n}\right) \in U$, if $x_{n} \neq 0$, then $\frac{\partial F_{n}}{\partial x_{n}}\left(x_{1}, \ldots, x_{n}\right)>0$. If this, last change of $U$ affects the genericity of $(a, 0)$, we replace $(a, 0)$ with another generic point and continue to assume that $(a, 0)$ is generic in $D$.

We define the map $\Psi: U \rightarrow \mathbf{R}^{n}$ by

$$
\Psi\left(x_{1}, \ldots, x_{n}\right)=\left(x_{1}, \ldots, x_{n-1}, F_{n}\left(x_{1}, \ldots, x_{n}\right)\right) .
$$

Claim. $\Psi$ is continuous, injective on $U$, the identity on $D$, differentiable on $U \backslash D$, and $\left|J(\Psi)_{x}\right|>0$ for all $x \in U \backslash D$.

Proof of Claim. Obviously $\Psi$ is continuous and differentiable on $U \backslash D$. It is injective since for every $\left(x_{1}, \ldots, x_{n-1}\right) \in D$ the function $x_{n} \mapsto F_{n}\left(x_{1}, \ldots, x_{n}\right)$ is strictly increasing.

We have $\left|J(\Psi)_{x}\right|=\frac{\partial F_{n}}{\partial x_{n}}(x)$, hence it is positive outside of $D$.

The above claim allows us to replace the map $F$ with the map $F \circ \Psi^{-1}$ without changing the sign of the Jacobean matrix of $F$ on $U \backslash D$. We therefore may assume from now on that $F_{n}\left(x_{1}, \ldots, x_{n}\right)=x_{n}$ on $U$. 
For $x=\left(x_{1}, \ldots, x_{n}\right) \in U \backslash D$ we have

$$
\begin{aligned}
\left|J(F)_{x}\right|=\left|\begin{array}{cccc}
\frac{\partial F_{1}}{\partial x_{1}}(x) & \cdots & \frac{\partial F_{1}}{\partial x_{n-1}}(x) & \frac{\partial F_{1}}{\partial x_{n}}(x) \\
\vdots & \ddots & \vdots & \vdots \\
\frac{\partial F_{n-1}}{\partial x_{1}}(x) & \cdots & \frac{\partial F_{n-1}}{\partial x_{n-1}}(x) & \frac{\partial F_{n-1}}{\partial x_{n}}(x) \\
0 & \cdots & 0 & 1
\end{array}\right| \\
=\left|\begin{array}{ccc}
\frac{\partial F_{1}}{\partial x_{1}}(x) & \cdots & \frac{\partial F_{1}}{\partial x_{n-1}}(x) \\
\vdots & \ddots & \vdots \\
\frac{\partial F_{n-1}}{\partial x_{1}}(x) & \cdots & \frac{\partial F_{n-1}}{\partial x_{n-1}}(x)
\end{array}\right| .
\end{aligned}
$$

Let $W=\left\{y \in \mathbf{R}^{n-1}:(y, 0) \in D\right\}$, and $f: W \rightarrow \mathbf{R}^{n-1}$ be the map $y \mapsto$ $\left(F_{1}(y, 0), \ldots, F_{n-1}(y, 0)\right)$. It is not hard to see that $f$ is continuous and injective on $W$. Also, since $a$ is a generic point in $W, a$ is a regular point of $f$. Hence $\left|J(f)_{a}\right| \neq 0$.

By Lemma 3.1 for $1 \leqslant i, j \leqslant n-1$, the limit of $\frac{\partial F_{i}}{\partial x_{j}}(u)$, as $u$ approaches $(a, 0)$ from $U$, equals $\frac{\partial f_{j}}{\partial x_{j}}(a)$. It follows then from (3.4) that

$$
\lim _{u \rightarrow(a, 0), u \in U}\left|J(F)_{u}\right|=\left|J(f)_{a}\right| .
$$

Hence, for $u \in U$ close enough to $(a, 0)$, whether in $V_{1}$ or in $V_{2}$, the sign of $\left|J(F)_{u}\right|$ is the same as the sign of $\left|J(f)_{a}\right|$. In particular, the sign of $|J(F)|$ is the same in $V_{1}$ and in $V_{2}$.

Example 3.3. To illustrate the above theorem, we consider a very simple example. Let $F: \mathbb{R} \rightarrow \mathbb{R}$ be defined as $x \mapsto \sqrt[3]{x}$. This is a semi-algebraic homeomorphism with $\operatorname{Reg}(F)$ consisting of two connected components, $\mathbb{R}^{>0}$ and $\mathbb{R}^{<0}$. Even though the function $F$ is not differentiable at 0 , the above theorem guarantees that the sign of the differential of $F$ to the right of 0 is the same as to the left.

3.2. Orientable definable manifolds. An orientation for definable manifolds was developed by A. Berarducci and M. Otero in 2] using local homology groups. In this section we use Theorem 3.2 to give an alternative definition that does not use homological methods. It resembles the definition of orientation for definable smooth manifolds, and indeed, in the case of definable $C^{2}$-manifolds our definition coincides with the corresponding definition in [1.

Definition 3.4. Let $U \subseteq \mathbf{R}^{n}$ be an open definable set and $f: U \rightarrow \mathbf{R}^{n}$ a definable continuous injective map. The map $f$ is orientation preserving if $\left|J(f)_{a}\right|>0$ for every $a \in \operatorname{Reg}(f)$, and it is orientation reversing if $\left|J(f)_{a}\right|<0$ for every $a \in \operatorname{Reg}(f)$.

Remark 3.5. If $U \subseteq \mathbf{R}^{n}$ is an open definable set that is definably connected, then, by Theorem 3.2. every definable injective $f: U \rightarrow \mathbf{R}^{n}$ is either orientation preserving or reversing. However, if $U$ is not definably connected, then $f$ may be neither orientation preserving nor reversing.

Let $M$ be a definable manifold of dimension $n$. Recall that a definable chart on $M$ is a pair $\langle U, \varphi\rangle$, where $U$ is a definable open subset of $M$ and $\varphi: U \rightarrow \mathbf{R}^{n}$ is a definable homeomorphism between $U$ and $f(U)$. A definable atlas on $M$ is a finite set $\mathcal{A}=\left\{\left\langle U_{i}, \varphi_{i}\right\rangle: i \in I\right\}$ of definable charts on $M$ such that $M=\bigcup_{i \in I} U_{i}$. 
Definition 3.6. A definable oriented manifold $\langle M, \mathcal{A}\rangle$ is a definable manifold $M$ together with a definable atlas $\mathcal{A}=\left\{\left\langle U_{i}, \varphi_{i}\right\rangle: i \in I\right\}$ such that all transition maps $\varphi_{i} \circ \varphi_{j}^{-1}$ are orientation preserving.

A definable manifold $M$ is called orientable if there is a definable atlas $\mathcal{A}$ on $M$ such that $\langle M, \mathcal{A}\rangle$ is a definable oriented manifold.

Let $\langle M, \mathcal{A}\rangle,\langle N, \mathcal{B}\rangle$ be definable oriented manifolds of the same dimension and $f: M \rightarrow N$ a definable continuous injective map. We say that $f$ is orientation preserving (orientation reversing) if for all charts $\langle U, \varphi\rangle \in \mathcal{A},\langle V, \psi\rangle \in \mathcal{B}$ the map $\psi \circ f \circ \varphi^{-1}$ is orientation preserving (orientation reversing).

Let $\langle M, \mathcal{A}\rangle$ be a definable oriented manifold and $V$ a definable open subset of $M$. It is easy to see that $\mathcal{A}\lceil V=\{\langle U \cap V, \varphi\lceil V\rangle:\langle U, \varphi\rangle \in \mathcal{A}, U \cap V \neq \varnothing\}$ is a definable atlas on $V$, and $\langle V, \mathcal{A} \mid V\rangle$ is a definable oriented manifold; this is called the induced orientation on $V$.

The following corollary follows from Theorem 3.2

Corollary 3.7. Let $\langle M, \mathcal{A}\rangle,\langle N, \mathcal{B}\rangle$ be definable oriented manifolds of the same dimension and $f: M \rightarrow N$ a definable continuous injective map. If $M$ is definably connected, then $f$ is either orientation preserving or orientation reversing.

Let $M$ be a definable manifold and $\mathcal{A}, \mathcal{B}$ two definable atlases on $M$ such that both $\langle M, \mathcal{A}\rangle$ and $\langle M, \mathcal{B}\rangle$ are definable oriented manifolds. We say that the orientations given by $\mathcal{A}$ and $\mathcal{B}$ are equivalent if the identity map id: $\langle M, \mathcal{A}\rangle \rightarrow\langle M, \mathcal{B}\rangle$ is orientation preserving; and we say that the orientations given by $\mathcal{A}$ and $\mathcal{B}$ are opposite if the identity map id: $\langle M, \mathcal{A}\rangle \rightarrow\langle M, \mathcal{B}\rangle$ is orientation reversing. It follows from Corollary 3.7 that for a definably connected orientable definable manifold, up to equivalence, there are exactly two definable orientations.

The following lemma provides a useful criterion when a definable manifold is orientable.

Lemma 3.8. Let $M$ be a definable manifold and $\mathcal{B}=\left\{\left\langle U_{i}, \varphi_{i}\right\rangle: i \in I\right\}$ a definable atlas on $M$ such that every $U_{i}$ is definably connected. Then $M$ is definably orientable if and only if there is a function $\lambda: I \rightarrow\{-1,+1\}$ such that $\operatorname{Sign}\left(\left|J\left(\varphi_{i} \circ \varphi_{j}^{-1}\right)\right|_{a}\right)=$ $\lambda(i) \lambda(j)$ for all $i, j \in I$ and every generic $a \in \varphi_{j}\left(U_{i} \cap U_{j}\right)$.

Proof. Assume $\mathcal{A}$ is a definable atlas on $M$ such that $\langle M, \mathcal{A}\rangle$ is definably oriented. The required function $\lambda$ can be obtained as follows. For $i \in I$ we assign $\lambda(i)$ to be +1 if the map $\varphi_{i}: U_{i} \rightarrow \mathbf{R}^{n}$ is orientation preserving and -1 if it is orientation reversing, with the orientation on $U_{i}$ induced from $\langle M, \mathcal{A}\rangle$.

For the opposite direction, assume that $\mathcal{B}$ and $\lambda$ are as in the statement. For $i \in I$ let $\hat{\varphi}_{i}$ be the map from $U_{i}$ into $\mathbf{R}^{n}$ that equals $\varphi_{i}$ if $\lambda(i)=+1$ and $r \circ \varphi_{i}$ if $\lambda(i)=-1$, where $r: \mathbf{R}^{n} \rightarrow \mathbf{R}^{n}$ is the reflection in the hyperplane $x_{n}=0$. It is not hard to see that $M$, with the definable atlas $\mathcal{A}=\left\{\left\langle U_{i}, \hat{\varphi}_{i}\right\rangle: i \in I\right\}$, is a definably oriented manifold.

3.3. Orientation on polyhedra. If $M$ is a definable manifold, then, by the Triangulation Theorem [4, Chapter 8], $M$ is definably homeomorphic to a simplicial complex. In this section we show that our notion of orientation coincides with a combinatorial orientation for simplicial complexes. In [2] it was also shown that in the case of definably compact manifolds, the combinatorial orientation coincides with the orientation given via local homology groups. It follows then that at least 
in the case of definably compact manifolds all of these three notions of orientation coincide.

We review briefly simplices and simplicial complexes. We refer to [4, Chapter 8] for more details.

3.3.1. Simplices and their orientations. An n-simplex $\sigma$ in $\mathbf{R}^{k}$ is a set spanned by $n+1$ affine independent points $a_{0}, \ldots, a_{n} \in \mathbf{R}^{k}$, i.e., the set $\left\{a_{i}-a_{0}: 1 \leqslant i \leqslant n\right\}$ is linearly independent and

$$
\sigma=\left\{\sum t_{i} a_{i}: t_{i}>0, \sum t_{i}=1\right\} .
$$

We will use $\triangle\left\{a_{0}, \ldots, a_{n}\right\}$ to denote the $n$-simplex spaned by $a_{0}, \ldots, a_{n}$.

$A$ face of $\triangle\left\{a_{0}, \ldots, a_{n}\right\}$ is a simplex spanned by a nonempty subset of $\left\{a_{0}, \ldots, a_{n}\right\}$.

Let $\sigma$ be an $n$-simplex and $\mathcal{O}(\sigma)$ the set of all orderings of vertices of $\sigma$. $A$ (combinatorial) orientation on $\sigma$ is a map $\mathfrak{o}: \mathcal{O}(\sigma) \rightarrow\{+1,-1\}$ that changes sign under a transposition of a pair of elements in an ordering. Obviously, to define an orientation on an $n$-simplex $\triangle\left\{a_{0}, \ldots, a_{n}\right\}$ it is sufficient to assign a sign to one of the orderings of $\left\{a_{0}, \ldots, a_{n}\right\}$.

Let $\sigma=\triangle\left\{a_{0}, \ldots, a_{n}\right\}, \sigma_{1}=\triangle\left\{b_{0}, \ldots, b_{n}\right\}$ be oriented $n$-simplexes with orientations $\mathfrak{o}, \mathfrak{o}_{1}$ respectively, and $f: \sigma \rightarrow \sigma_{1}$ the simplicial isomorphism induced by the vertex map $a_{i} \mapsto b_{i}$. We say that $f$ is orientation preserving, if $\mathfrak{o}\left(a_{0}, \ldots, a_{n}\right)=$ $\mathfrak{o}_{1}\left(b_{0}, \ldots, b_{n}\right)$; otherwise we say that it is orientation reversing.

If $\sigma=\triangle\left\{a_{0}, \ldots, a_{n}\right\}$ is an $n$-simplex in $\mathbf{R}^{n}$, then it inherits an orientation from $\mathbf{R}^{n}$ by assigning to $\left(a_{0}, \ldots, a_{n}\right)$ the sign of the determinant of the matrix whose columns are the vectors $\left(a_{1}-a_{0}, \ldots, a_{n}-a_{0}\right)$. We will call this orientation the induced orientation on $\sigma$.

If $\sigma$ is an $n$-simplex, then a map $i: \sigma \rightarrow \mathbf{R}^{n}$ is called a simplicial embedding if $i$ is a simplicial isomorphism from $\sigma$ onto an $n$-simplex in $\mathbf{R}^{n}$. If $\sigma$ is an oriented $n$-simplex and $i: \sigma \rightarrow \mathbf{R}^{n}$ is a simplicial embedding, then, considering $i(\sigma)$ with the orientation induced from $\mathbf{R}^{n}$, we say that $i$ is an orientation preserving (reversing) embedding if it is an orientation preserving (reversing) simplicial isomorphism.

The following claim is elementary.

Claim 3.9. Let $\sigma, \sigma^{\prime}$ be $n$-simplices in $\mathbf{R}^{n}$ with induced orientations and $f: \sigma \rightarrow \sigma^{\prime}$ a simplicial isomorphism. Then $f$ is orientation preserving, as a simplicial map, if and only if $f$ preserves orientation as a map from an open subset of $\mathbf{R}^{n}$ into $\mathbf{R}^{n}$.

Let $\sigma$ be an oriented $n$-simplex, $U$ an open subset of $\mathbf{R}^{n}$, and $f: U \rightarrow \sigma$ a definable continuous injective map. We say that $f$ is orientation preserving (reversing) if for some orientation preserving simplicial embedding $i: \sigma \rightarrow \mathbf{R}^{n}$ the map $i \circ f$ is orientation preserving (reversing), as a map from an open set $U$ to $\mathbf{R}^{n}$. It follows from Claim 3.9 that this definition does not depend on the choice of $i$. If $U$ is definably connected, then every definable continuous injective map from $U$ into $\sigma$ is either orientation preserving or reversing.

3.3.2. Contiguous simplices. Two disjoint $n$-simplices $\sigma, \sigma_{1}$ are called contiguous if they share a common $(n-1)$-face.

Let $\sigma=\triangle\left\{b, a_{1}, \ldots, a_{n}\right\}, \sigma_{1}=\triangle\left\{c, a_{1}, \ldots, a_{n}\right\}$ be contiguous $n$-simplices with orientations $\mathfrak{o}, \mathfrak{o}_{1}$, respectively. We say that $\mathfrak{o}$ and $\mathfrak{o}_{1}$ are compatible if $\mathfrak{o}\left(b, a_{1}, \ldots, a_{n}\right)$ $=-\mathfrak{o}\left(c, a_{1}, \ldots, a_{n}\right)$. 
Claim 3.10. Let $\sigma, \sigma_{1}$ be contiguous $n$ simplices in $\mathbf{R}^{n}$. Then the induced orientations are compatible.

Proof. Easy.

3.3.3. Simplicial complexes and their orientation. A coherent orientation on an $n$-dimensional simplicial complex $K$ is a choice of an orientation $\mathfrak{o}_{\sigma}$ for each $n$ simplex $\sigma$ in $K$ such that contiguous $n$-simplices have compatible orientations. (In particular, each $(n-1)$-simplex in $K$ is a face of at most two $n$-simplices in $K$.) If $K$ has a coherent orientation, then it is called orientable.

It follows from Claim 3.10 that if $K$ is an $n$-dimensional simplicial complex in $\mathbf{R}^{n}$, then the induced orientation on every $n$-simplex in $K$ gives a coherent orientation on $K$.

A simplicial complex $K$ of dimension $n$ is said to be strongly connected if the graph $\Gamma$, whose vertices are $n$-simplices of $K$ with edges connecting contiguous simplices, is connected.

As usual, if $K$ is a simplicial complex in $\mathbf{R}^{k}$, then by $|K|$ we will denote the polyhedron in $\mathbf{R}^{k}$ spanned by $K$, i.e., $|K|=\bigcup_{\sigma \in K} \sigma$.

Claim 3.11. If $\sigma_{1}$ and $\sigma_{2}$ are contiguous $n$-simplices with common $(n-1)$-face $\sigma^{\prime}$, then there is a simplicial isomorphism $i^{*}$ sending the simplicial complex $\left\{\sigma_{1}, \sigma_{2}, \sigma^{\prime}\right\}$ onto a complex in $\mathbf{R}^{n}$. Moreover, if $\sigma_{1}$ and $\sigma_{2}$ are equipped with compatible orientations, then we can choose $i^{*}$ to be orientation preserving on each $\sigma_{1}$ and $\sigma_{2}$. (As usual, we use orientation induced from $\mathbf{R}^{n}$ on the image of $i^{*}$.)

Proof. Easy.

Let $K$ be an oriented $n$-dimensional simplicial complex, $U$ an open subset of $\mathbf{R}^{n}$ and $f: U \rightarrow|K|$ a definable continuous injective map. We say that $f$ is orientation preserving (reversing) if for each $n$-simplex $\sigma \in K$ with $f(U) \cap \sigma \neq \varnothing$ the map $f$, restricted to $f^{-1}(\sigma)$, is orientation preserving (reversing), as a map from an open subset of $\mathbf{R}^{n}$ into an oriented $n$-simplex.

Lemma 3.12. Assume $|K|$ is the $n$-dimensional polyhedron in $\mathbf{R}^{k}$ spanned by an oriented simplicial complex $K, U$ a definably connected open subset of $\mathbf{R}^{n}$, and $f: U \rightarrow|K|$ a definable continuous injective map. Then $f$ is either orientation preserving or orientation reversing.

Proof. We consider all $n$-simplices $\sigma \in K$ with $f(U) \cap \sigma \neq \varnothing$, and for each such $\sigma$ we consider the connected components of $U \cap f^{-1}(\sigma)$. Thus we can find in $U$ finitely many definably connected open sets $V_{1}, \ldots, V_{l}$ such that:

(1) For each $V_{r}$, there is an $n$-simplex $\sigma_{r} \in K$ such that $f\left(V_{r}\right)$ is contained in $\sigma_{r}$.

(2) The set $\left(V_{1} \cup \cdots \cup V_{l}\right)$ is dense in $U$.

By Fact 2.2, it is sufficient to show that if $\operatorname{dim}\left(\operatorname{cl}\left(V_{r}\right) \cap \operatorname{cl}\left(V_{s}\right)\right)=n-1$ and $f\left\lceil V_{r}\right.$ is orientation preserving, then $f \nmid V_{s}$ is orientation preserving as well. We fix such $V_{r}$, $V_{s}$ and assume $f\left\lceil V_{r}\right.$ is orientation preserving. Since $\operatorname{dim}\left(\operatorname{cl}\left(V_{r}\right) \cap \operatorname{cl}\left(V_{s}\right)\right)=n-1$, there is a definable subset $D \subseteq\left(\operatorname{cl}\left(V_{r}\right) \cap \operatorname{cl}\left(V_{s}\right)\right)$ such that $f(D)$ is an open subset of an $(n-1)$-simplex $\sigma^{\prime}$ of $K$. Obviously, $\sigma^{\prime}$ is a common face of $\sigma_{r}, \sigma_{s}$. Hence $\sigma_{r}, \sigma_{s}$ are contiguous, and the set $W=V_{r} \cup D \cup V_{s}$ is a definably connected open subset of $U$.

By Claim 3.11 we can find a simplicial isomorphism $i^{*}$ from $\left\{\sigma_{r}, \sigma_{s}, \sigma^{\prime}\right\}$ onto a simplicial complex in $\mathbf{R}^{n}$ that preserves orientation on each $\sigma_{r}$ and $\sigma_{s}$. 
Since $W$ is a definable connected open subset of $\mathbf{R}^{n}$, the map $i^{*} \circ f$ restricted to $W$ is either orientation preserving or orientation reversing. Its restriction to $V_{r}$ is orientation preserving, hence the restriction to $V_{s}$ must be orientation preserving as well.

Theorem 3.13. Assume that $M$ is a definably connected definable manifold, definably homeomorphic to a polyhedron $|K| \subseteq \mathbf{R}^{k}$ spanned by a complex $K$. Then every orientation on $M$ induces a coherent orientation on $K$ and vice versa.

Proof. Let $n$ be the dimension of $M$. First note that since $M$ is a definably connected manifold, the complex $K$ is strongly connected. Let $F: M \rightarrow|K|$ be a definable homeomorphism.

Assume first that $K$ is equipped with a coherent orientation. We are going to use Lemma 3.8 to produce a definable orientation on $M$.

Let $\mathcal{B}=\left\{\left\langle U_{i}, \varphi_{i}\right\rangle: i \in I\right\}$ be a definable atlas on $M$ with all $U_{i}$ definably connected. We need to construct a function $\lambda: I \rightarrow\{-1,+1\}$ as in Lemma 3.8.

Let $i \in I$. By Lemma 3.12, the map $F \circ \varphi_{i}^{-1}$ is either orientation preserving or orientation reversing, as a map from $\varphi_{i}(U)$ into $|K|$. We assign $\lambda(i)=+1$ if $F \circ \varphi_{i}^{-1}$ is orientation preserving and $\lambda(i)=-1$ otherwise.

It remains to verify that $\lambda$ satisfies the assumptions of Lemma 3.8. Assume that $a$ is a generic point in $\varphi_{i}\left(U_{i} \cap U_{j}\right)$. Let $\sigma$ be the $n$-simplex in $K$ with $F(a) \in \sigma$, and $i: \sigma \rightarrow \mathbf{R}^{n}$ an orientation preserving simplicial embedding. We have

$$
\lambda(i)=\operatorname{Sign}\left(\left|J\left(i \circ F \circ \varphi_{i}^{-1}\right)_{a}\right|\right) \quad \text { and } \lambda(j)=\operatorname{Sign}\left|J\left(i \circ F \circ \varphi_{j}^{-1}\right)_{a}\right| .
$$

It follows immediately that $\lambda(i) \lambda(j)=\operatorname{Sign}\left(\left|J\left(\varphi_{i} \circ \varphi_{j}^{-1}\right)_{a}\right|\right)$.

We now need to prove the converse, namely, that any orientation on the manifold $M$ induces a coherent orientation on $K$. This is done as follows: For each $n$ simplex $\sigma \in K$, consider the restriction of $F$ to the oriented, definably connected manifold $M_{\sigma}=F^{-1}(\sigma)$. We choose a simplicial embedding $i_{\sigma}: \sigma \rightarrow \mathbf{R}^{n}$ so that $i_{\sigma} \circ F: M_{\sigma} \rightarrow R^{n}$ preserves orientation. We orient $\sigma$ by transferring via $i$ the orientation induced by $\mathbf{R}^{n}$ on $i_{\sigma}(\sigma)$. We need to show that this orientation is a coherent orientation on the whole of $K$.

Consider contiguous $n$-simplices, $\sigma$ and $\sigma_{1}$ in $K$ with an $(n-1)$-simplex $\sigma^{\prime}$ as a common face. By definition, the maps $i_{\sigma} \circ F$ and $i_{\sigma_{1}} \circ F$ preserve orientation on $M_{\sigma}$ and $M_{\sigma_{1}}$, respectively.

Let $i^{*}$ be a map from the simplicial complex $\left\{\sigma, \sigma_{1}, \sigma^{\prime}\right\}$ onto a simplicial complex in $\mathbf{R}^{n}$ as in Claim 3.10. Modifying $i^{*}$ if needed, we may assume that $i^{*} \circ F$ is orientation preserving on $F^{-1}\left(\sigma \cup \sigma_{1} \cup \sigma^{\prime}\right)$.

But then, both maps $i^{*} \circ i_{\sigma}^{-1}$ and $i^{*} \circ i_{\sigma_{1}}^{-1}$ preserve orientations, as maps from open subsets of $\mathbf{R}^{n}$ into $\mathbf{R}^{n}$. It follows then that the combinatorial orientations of $\sigma$ and $\sigma_{1}$ agree with $i^{*}(\sigma)$ and $i^{*}\left(\sigma_{1}\right)$, respectively. Since the complex $i^{*}\left(\sigma \cup \sigma_{1} \cup \sigma^{\prime}\right)$ inherits from $\mathbf{R}^{n}$ a coherent orientation, the orientation on $\sigma \cup \sigma_{1} \cup \sigma^{\prime}$ is coherent as well.

3.4. Degree of a map. A degree of a definable continuous map was defined by M. Edmundo and M. Otero in [6] using homological methods. In this section we will give an alternative definition that does not use homology.

Definition 3.14. Let $\langle M, \mathcal{A}\rangle$ and $\langle N, \mathcal{B}\rangle$ be definable oriented manifolds of the same dimension and $f: M \rightarrow N$ a definable continuous map. Let $a \in M$ be such 
that $f$ is injective at $a$. Then the local degree of $f$ at $a$, denoted by $\operatorname{deg}_{a} f$, is defined to be +1 if $f\lceil U: U \rightarrow N$ is orientation preserving on an open neighborhood of $a$ (with respect to the induced orientation), and $\operatorname{deg}_{a} f=-1$ if it is orientation reversing.

Let $f:\langle M, \mathcal{A}\rangle \rightarrow\langle N, \mathcal{B}\rangle$ be a definable continuous map between two definable oriented manifolds of the same dimension and $b \in N$. We say that $b$ is a topological regular value of $f$ if $f^{-1}(b)$ is finite (or empty), and $f$ is locally injective at every $a \in f^{-1}(b)$. If $b$ is a topological regular value of $f$, then we will denote by $\operatorname{deg}^{b}(f)$ the integer $\sum_{f(a)=b} \operatorname{deg}_{a}(f)$. (If $b$ is not in the image of $f$, then $\operatorname{deg}^{b}(f)=0$.)

Notice that if $f: M \rightarrow N$ is a definable continuous map between two manifolds of the same dimension, then every generic $b \in N$ is a topological regular value of $f$. In particular, the set of topologically regular values is a dense subset of $N$.

Recall that a definable continuous map $f: M \rightarrow N$ between definable manifolds is called definably proper if the preimage $f^{-1}(S)$ of every definably compact subset $S \subseteq N$ is definably compact.

Claim 3.15. Let $f: M \rightarrow N$ be a definable continuous map between definable manifolds.

(1) If $M$ is definably compact, then $f$ is definably proper.

(2) If $f$ is definably proper, then $f(M)$ is a closed subset of $N$.

(3) If $f$ is definably proper and $A \subseteq N$ a definable open set, then $f \uparrow f^{-1}(A)$ is definably proper (as a map from $f^{-1}(A)$ into $A$ ).

Proof. Easy.

The next theorem is the main goal of this section.

Theorem 3.16. Let $\langle M, \mathcal{A}\rangle,\langle N, \mathcal{B}\rangle$ be definably oriented manifolds of the same dimension $n$, and $f: M \rightarrow N$ a definable continuous map which is definably proper. Assume $N$ is definably connected. Then $\operatorname{deg}^{y}(f)$ is the same for all topological regular values $y \in N$.

Proof. Let $V^{r e g} \subseteq N$ be the set of all topological regular values of $f$.

Claim. $V^{\text {reg }}$ is definable, open, $\operatorname{dim}\left(N \backslash V^{r e g}\right)<n$, and the function $y \mapsto$ $\operatorname{deg}^{y}(f)$ is locally constant on $V^{\text {reg }}$.

Proof of Claim. Definability of $V^{\text {reg }}$ is easy. Since $V^{\text {reg }}$ contains all generic points of $N, \operatorname{dim}\left(N \backslash V^{r e g}\right)<n$.

Let $b \in V^{r e g}$. We need to find an open definable $W \subseteq N$ containing $b$ such that $W \subseteq V^{r e g}$ and $y \mapsto \operatorname{deg}^{y}(f)$ is constant on $W$.

Case 1: $b \notin$ Image $(f)$.

Since $f$ is definably proper, the image of $f$ is closed in $N$ and we can take $W=N \backslash$ Image $(f)$.

Case 2: $b \in \operatorname{Image}(f)$.

Let $a_{1}, \ldots, a_{k}$ be all preimages of $b$ under $f$, and let $U_{1}, \ldots, U_{k}$ be open definable pairwise disjoint subsets of $M$ such that $a_{i} \in U_{i}$ and $f$ is injective on each $U_{i}$. By the Invariance of Domain Theorem, every $f\left(U_{i}\right)$ is open in $N$. Since $f$ is definably proper, there is a definably connected open neighborhood $W$ of $b$ such that $W \subseteq f\left(U_{i}\right)$ for each $i$. Obviously, the function $y \mapsto \operatorname{deg}^{y}(f)$ is constant on $W$.

For a tuple $x \in \mathbf{R}^{n}$ we will denote by $x_{n}$ the $n$th component of $x$. 
We first consider a special case.

A special case. Let us assume in addition the following:

(a1) $N=\mathbf{R}^{n}$ with the standard orientation;

(a2) every $y \in \mathbf{R}^{n}$ with $y_{n} \neq 0$ is a topological regular value of $f$;

(a3) $\left\{y \in \mathbf{R}^{n}: y_{n}>0\right\} \subseteq$ Image $(f)$;

(a4) Image $(f) \subseteq\left\{y \in \mathbf{R}^{n}: y_{n} \geqslant 0\right\}$;

(a5) $M=\mathbf{R}^{n}$ with the standard orientation;

(a6) $\pi \circ f=\pi$, where $\pi: \mathbf{R}^{n} \rightarrow \mathbf{R}^{n-1}$ is the projection onto the first $n-1$ coordinates.

Proof of the special case. In order to prove the theorem in this case, it is sufficient to show that $\operatorname{deg}^{y}(f)=0$ for all topological regular values $y$ of $f$.

Since $f$ is definably proper, its image is closed in $N$, hence Image $(f)=\{y \in$ $\left.\mathbf{R}^{n}: y_{n} \geqslant 0\right\}$.

If $y \in \mathbf{R}^{n}$ with $y_{n}=0$, then $y$ belongs to the frontier of $f(M)$, hence, by the Invariance of Domain Theorem, it cannot be a topological regular value. Thus the set of topological regular values of $f$ is $\left\{y \in \mathbf{R}^{n}: y_{n} \neq 0\right\}$. It has two definably connected components $\left\{y_{n}>0\right\}$ and $\left\{y_{n}<0\right\}$, and $\operatorname{deg}^{y}(f)=0$ for all $y \in$ $\left\{y_{n}<0\right\}$. To finish this special case we need to show that $\operatorname{deg}^{y}(f)=0$ for some $y \in\left\{y_{n}>0\right\}$.

Let $c \in \mathbf{R}^{n-1}$ be arbitrary. According to assumptions (a6) and (a4), $f(\{c\} \times \mathbf{R}) \subseteq$ $\{c\} \times \mathbf{R} \geqslant 0$. Let $h_{c}: \mathbf{R} \rightarrow \mathbf{R}^{\geqslant 0}$ be the function such that $f((c, t))=\left(c, h_{c}(t)\right)$. Since $f$ is definably proper, we obtain that $I_{0}=h_{c}^{-1}(0)$ is a definably compact subset of $\mathbf{R}$, so it consists of finitely many closed intervals of $\mathbf{R}$ (we treat points as closed intervals). If $t_{0} \notin I_{0}$, then $f\left(\left(c, t_{0}\right)\right)$ is a topological regular value of $f$, hence $f$ is locally injective at $\left(c, t_{0}\right)$ and $h_{c}(t)$ must be strictly monotone near $t_{0}$. Since $h_{c}(t)$ is continuous, it implies that $h_{c}(t)$ is strictly monotone outside of $I_{0}$. By (a4), $h_{c}$ has only nonnegative values, hence $I_{0}$ must be an interval $\left[\alpha_{c}^{-}, \alpha_{c}^{+}\right]$, and $h_{c}(t)$ has the following form: It is strictly decreasing on $\left(-\infty, \alpha_{c}^{-}\right)$, zero on $\left[\alpha_{c}^{-}, \alpha_{c}^{+}\right]$, and strictly increasing on $\left(\alpha_{c}^{+},+\infty\right)$.

Notice also that, since $f$ is definably proper, the $\operatorname{limit} \lim h_{c}(t)$ does not exist in $\mathbf{R}$, as $t$ goes to $+\infty$ or $-\infty$. Therefore this limit must be $+\infty$.

Thus for any positive $t_{0} \in \mathbf{R}$ the preimage of the point $p=\left(c, t^{0}\right)$ under $f$ consists of two points: $p^{+}=\left(c, t_{0}^{+}\right)$with $t_{0}^{+}>a_{c}^{+}$, and $p^{-}=\left(c, t_{0}^{-}\right)$with $t_{0}^{-}<a_{c}^{-}$. The function $h_{c}(t)$ is increasing at $t_{0}^{+}$and decreasing at $t_{0}^{-}$. Since the map $f\left(x_{1}, \ldots, x_{n}\right)$ has the form

$$
\left(x_{1}, \ldots, x_{n}\right) \mapsto\left(x_{1}, \ldots, x_{n-1}, h_{\left(x_{1}, \ldots, x_{n-1}\right)}\left(x_{n}\right)\right),
$$

it is easy to see that $\operatorname{deg}_{p^{-}}(f)=-1, \operatorname{deg}_{p^{+}}(f)=+1$, and $\operatorname{deg}^{p}(f)=0$.

We now return to the general case, and reduce it to the special case above.

Let $V_{1}, \ldots, V_{k}$ be all definably connected components of $V^{r e g}$. By the claim above, the function $y \mapsto \operatorname{deg}^{y}(f)$ is constant on each $V_{i}$. Let $d_{i}$ be the value of this function on the component $V_{i}$. By Fact 2.2, we need to show that $d_{i}=d_{j}$ for $V_{i}, V_{j}$ with $\operatorname{dim}\left(\operatorname{cl}\left(V_{i}\right) \cap \operatorname{cl}\left(V_{j}\right)\right)=n-1$.

We assume that $V, V^{\prime}$ are two such components, and let $D=\operatorname{cl}(V) \cap \operatorname{cl}\left(V^{\prime}\right)$. We need to show that $\operatorname{deg}^{y}(f)=\operatorname{deg}^{y_{1}}(f)$ for some $y \in V, y_{1} \in V^{\prime}$. If both $V$ and $V^{\prime}$ are not in the image of $f$, then both degrees are zeroes and there is nothing to prove. Thus we may assume that $V \subseteq \operatorname{Image}(f)$.

By o-minimality, outside of a set of smaller dimension, the set $D$ is a submanifold of $N$. Hence we can find an open definable set $B \subseteq N$ and an orientation preserving 
homeomorphism $\lambda$ from $B$ onto $\mathbf{R}^{n}$ such that $\lambda(B \cap D)=\left\{y \in \mathbf{R}^{n}: y_{n}=0\right\}$. We may assume that $\lambda(B \cap V)=\left\{y \in \mathbf{R}^{n}: y_{n}>0\right\}$ and $\lambda\left(B \cap V^{\prime}\right)=\left\{y \in \mathbf{R}^{n}: y_{n}<0\right\}$. Replacing the manifold $N$ with $\mathbf{R}^{n}, M$ with $f^{-1}(B)$, and the map $f$ with $\lambda \circ f$ we may assume that the conditions (a1)-(a2) hold.

To obtain the condition (a3), we compose $f$ with the following map: Let $\sigma: \mathbf{R}^{n} \rightarrow$ $\mathbf{R}^{n}$ be the mapping

$$
\left(y_{1}, \ldots, y_{n-1}, y_{n}\right) \mapsto\left(y_{1}, \ldots, y_{n-1},\left|y_{n}\right|\right) .
$$

It is easy to see that $\sigma$ is a continuous proper map injective on each half-space $\{y \in$ $\left.\mathbf{R}^{n}: y_{n}>0\right\}$ and $\left\{y \in \mathbf{R}^{n}: y_{n}<0\right\}$. It is orientation preserving on $\left\{y_{n}>0\right\}$ and orientation reversing on $\left\{y_{n}<0\right\}$. Consider the map $f_{1}=\sigma \circ f$. If $y=\left(y_{1}, \ldots, y_{n}\right) \in$ $\left\{y_{n}>0\right\}$, then $\operatorname{deg}^{y}\left(f_{1}\right)=\operatorname{deg}^{y}(f)-\operatorname{deg}^{\tilde{y}}(f)$, where $\tilde{y}=\left(y_{1}, \ldots,-y_{n}\right)$. Hence, to prove the theorem it is sufficient to show that $\operatorname{deg}^{y}\left(f_{1}\right)=0$ for some $y \in\left\{y_{n}>0\right\}$. Since the image of $\sigma$ is $\left\{y \in \mathbf{R}^{n}: y_{n} \geqslant 0\right\}$, we replace $f$ by $f_{1}$ if needed and assume that condition (a4) also holds.

We now turn to the conditions (a5) and (a6). Consider the map $g=\pi \circ f: M \rightarrow$ $\mathbf{R}^{n-1}$, where $\pi: \mathbf{R}^{n} \rightarrow \mathbf{R}^{n-1}$ is the projection onto the first $n-1$ coordinates. Since the image of $f$ contains the half-space $\left\{y \in \mathbf{R}^{n}: x_{n}>0\right\}$, the map $g$ is surjective. Obviously, it is definable and continuous. By the Trivialization Theorem [4, there is an open ball $B \subseteq \mathbf{R}^{n-1}$ such that $g$ is trivial over $B$, i.e., there is a definable set $J \subseteq \mathbf{R}^{k}$ and a definable homeomorphism $\nu: g^{-1}(B) \rightarrow B \times J$ such that the following diagram is commutative:

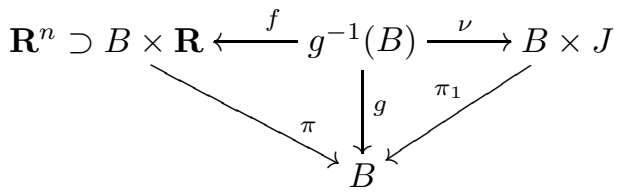

where $\pi_{1}: B \times J \rightarrow B$ is the natural projection.

We now replace $N$ with $B \times \mathbf{R}, M$ with $g^{-1}(B)$, and $f$ with $f\left\lceil g^{-1}(B)\right.$. After this replacement, conditions (a1)-(a4) are not satisfied anymore. However, the open ball $B$ is definably homeomorphic to $\mathbf{R}^{n-1}$. Therefore, after applying an appropriate homeomorphism, we can replace $B$ with $\mathbf{R}^{n-1}$, and assume that conditions (a1)-(a4) are satisfied, and we may also assume that the following diagram is commutative:

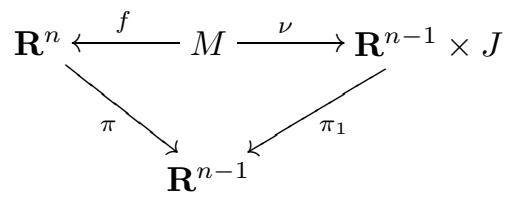

where $\nu: M \rightarrow \mathbf{R}^{n-1} \times J$ is a definable homeomorphism.

By Theorem 2.8, $J$ is a definable one-dimensional manifold. Let $J_{1}, \ldots, J_{k}$ be the definably connected components of $J$ and $M_{i}=\nu^{-1}\left(\mathbf{R}^{n-1} \times J_{i}\right), i=1, \ldots, k$. Since $\nu$ is a homeomorphism, $M_{i}, i=1, \ldots, k$, are the definably connected components of $M$. We will consider each $M_{i}$ as a definable oriented manifold with the orientation induced from $M$.

Let $f_{i}=f\left\lceil M_{i}\right.$. Clearly, $\operatorname{deg}^{y}(f)=\operatorname{deg}^{y}\left(f_{1}\right)+\cdots+\operatorname{deg}^{y}\left(f_{k}\right)$. Thus we can consider each $f_{i}$ separately and assume that $J$ is definably connected. 
Case 1: $J$ is definably compact.

Pick any $a \in \mathbf{R}^{n-1}$. Then $f(\{a\} \times J)$ is contained in $\{a\} \times \mathbf{R}$ and is definably compact, hence bounded. Thus $(a, t)$ is not in the image of $f$ for any sufficiently large positive $t$, therefore, $\operatorname{deg}^{(a, t)}(f)=0$ for any large enough $t>0$.

Case 2: $J$ is not definably compact.

In this case, by [17, Proposition 2], $J$ is homeomorphic to $\mathbf{R}$. Thus we may assume $J=\mathbf{R}$ and $M=\mathbf{R}^{n}$. This is exactly the setting of the special case. The theorem is proved.

The above theorem allows us to give a homology-free definition of the degree of a map.

Definition 3.17. Let $\langle M, \mathcal{A}\rangle,\langle N, \mathcal{B}\rangle$ be definable oriented manifolds of the same dimension and $f: M \rightarrow N$ a definable continuous definably proper map. If $N$ is definably connected, then we define the degree of $f$, denoted by $\operatorname{deg}(f)$, to be the integer $\operatorname{deg}^{a}(f)$, where $a \in N$ is any topologically regular value of $f$.

Claim 3.18. Let $\langle M, \mathcal{A}\rangle,\langle N, \mathcal{B}\rangle$ be definable oriented manifolds of the same dimension and $f: M \rightarrow N$ a definable continuous definably proper map. Assume $M$ is definably compact, $N$ is definably connected and not definably compact. Then $\operatorname{deg}(f)=0$.

Proof. Since $M$ is definably compact, $f(M)$ is also definably compact. Hence $f(M) \neq N, N \backslash f(M)$ is open and contains a topological regular value $a$. By the definition, $\operatorname{deg}(f)=\operatorname{deg}^{a}(f)=0$.

\subsection{Definable homotopies and the Brouwer fixed point theorem.}

3.5.1. Definable homotopy. Let $U, V$ be definable spaces, and $f_{0}, f_{1}: U \rightarrow V$ definable continuous maps. As usual, a definable homotopy from $f_{0}$ to $f_{1}$ is a definable continuous map $F: U \times[0,1] \rightarrow V$ such that $f_{0}(x)=F(x, 0)$ and $f_{1}(x)=F(x, 1)$. $A$ definable homotopy $F(x, t)$ from $f_{0}$ to $f_{1}$ is called proper if, in addition, $F$ is a definably proper map from $U \times[0,1]$ to $V$.

Theorem 3.19. Let $U, V$ be definably oriented manifolds of the same dimension with $V$ definably connected and $f_{0}, f_{1}: U \rightarrow V$ definably proper continuous maps. If $f_{0}$ and $f_{1}$ are homotopic via a proper definable homotopy, then they have the same degree.

Proof. Let $F: U \times[0,1]$ be a definable homotopy from $f_{0}$ to $f_{1}$ that is proper. We extend $F$ to a definable map from $U \times \mathbf{R}$ into $V$ by setting $F(u, t)=F(u, 0)$ for $t<0$ and $F(u, t)=F(u, 1)$ for $t>1$. Consider the map $H: U \times \mathbf{R} \rightarrow V \times \mathbf{R}$ given by

$$
H:(u, t) \mapsto(F(u, t), t) .
$$

It is not hard to see that $H$ is a definably proper continuous map. We orient $U \times \mathbf{R}$ and $V \times \mathbf{R}$ with product orientations. Let $a \in V$ be a generic point, and $t_{0}, t_{1} \in \mathbf{R}$ be such that each $t_{i}$ is generic over $a, t_{0}<0$ and $t_{1}>1$. Then $\left(a, t_{0}\right)$ and $\left(a, t_{1}\right)$ are topological regular values of $H$, and, by Theorem 3.16] we have that $\operatorname{deg}^{\left(a, t_{0}\right)}(H)=\operatorname{deg}^{\left(a, t_{1}\right)}(H)$. Since both $\left(a, t_{0}\right),\left(a, t_{1}\right)$ are generic points in $V \times \mathbf{R}$, they are regular values of $H$, the degrees can be computed as the sums of the signs 
of the corresponding Jacobians, and it is easy to see that for each $t_{0}, t_{1}$ the degree $\operatorname{deg}^{\left(a, t_{i}\right)}(H)$ is the same as the degree of the map $u \mapsto F\left(u, t_{i}\right)$.

3.5.2. Brouwer fixed point theorem. In this section we present a proof of a theorem that, as in the classical case, implies the Brouwer fixed point theorem that every definable continuous map from a closed ball in $\mathbf{R}^{n}$ into itself has a fixed point. In the o-minimal setting this theorem has already been proved by various methods (e.g., see [20], 22, 21]), however, we think that the proof presented here is a good illustration of the usefulness of our notion of degree.

We fix $n \in \mathbb{N}^{+}$. Let $\bar{B}$ be the unit closed ball in $\mathbf{R}^{n}, S$ the unit sphere, and $B$ the open unit ball $\bar{B} \backslash S$. We will consider $B$ as a definably oriented manifold, with the orientation induced from $\mathbf{R}^{n}$.

Recall that a definable continuous map $f: \bar{B} \rightarrow S$ is called a retraction if $f\lceil S=$ $\operatorname{id}_{S}$.

Theorem 3.20. There is no definable continuous retraction from $\bar{B}$ to $S$.

Proof. Towards getting a contradiction we assume that $f: \bar{B} \rightarrow S$ is a definable continuous retraction.

Consider the map $F: \bar{B} \times[0,1] \rightarrow \mathbf{R}^{n}$ given by

$$
F:(u, t) \mapsto t f(u)+(1-t) u .
$$

For each $r \in[0,1)$ the map $x \mapsto F(x, r)$ is identity on $S$ and maps $B$ into $B$.

Let $r \in[0,1)$ be fixed. It is not hard to see that the restriction of $F$ to $B \times[0, r]$ is a definably proper map, as a map into $B$. Thus the map $u \mapsto F(u, r)$ is homotopic (as a map from $B$ to $B$ ) to the identity map via a proper definable homotopy. By Theorem 3.19, its degree is 1 , hence its image is everywhere dense in $B$.

Thus, by the definable choice, there is a definable curve $\gamma(r):[0,1) \rightarrow B$ such that $\|F(\gamma(r), r)\| \leqslant 1 / 2$. Let $a$ be the limit of $\gamma(r)$ as $r$ approaches 1 . Obviously, $a \in$ $\bar{B}$ and $\|F(a, 1)\| \leqslant 1 / 2$. But $F(a, 1)=f(a)$ and $f(\bar{B}) \subseteq S$. A contradiction.

\section{Euler CHARACTERISTIC AND LefSCHETZ Number FOR SMOOTH MANifolds}

4.1. The gradient and the Hessian matrix of a function. Whenever we consider $\mathbf{R}^{n}$ as a vector space, we write the elements of $\mathbf{R}$ as column vectors. If $U \subseteq \mathbf{R}^{n}$ is a definable open set and $x \in U$, then the tangent space to $U$ at $x$ will always be identified with $\mathbf{R}^{n}$ in the usual way. Under this identification, if $f: \mathbf{R}^{n} \rightarrow \mathbf{R}^{m}$ is a definable $C^{1}$-function and $x \in \mathbf{R}^{n}$, then the differential of $\mathrm{D}(f)_{x}$ is a linear map from $\mathbf{R}^{n}$ to $\mathbf{R}^{m}$, and the Jacobian $J(f)_{x}$ is its matrix with respect to the standard coordinates.

Let $U \subseteq \mathbf{R}^{n}$ be an open definable set and $f: U \rightarrow \mathbf{R}$ a definable $C^{1}$-function. For $x \in U$ the Jacobian of $f$ at $x$ is an $1 \times n$-matrix, its transpose can be considered as an element of $\mathbf{R}^{n}$, and is called the gradient of $f$ at $x$. We will denote the gradient of $f$ at $x$ by $\nabla f(x)$. Thus $\nabla f(x)=\left(J(f)_{x}\right)^{T}$. If $\langle\cdot, \cdot\rangle$ is the standard inner product on $\mathbf{R}^{n}$, then for every $v \in \mathbf{R}^{n}$ we have $\mathrm{D}(f)_{x}(v)=J(f)_{x} v=\langle\nabla f(x), v\rangle$.

Let $U \subseteq \mathbf{R}^{n}$ be an open definable set and $f: U \rightarrow \mathbf{R}$ a definable $C^{2}$-function. The map $x \mapsto \nabla f(x)$ is a definable $C^{1}$-map from $U$ into $\mathbf{R}^{n}$ and, for $a \in U$, the Jacobian of this map at $a$ is called the Hessian matrix of $f$ at $a$. It is the square $n \times n$ matrix of the second order partial derivatives of $f$ computed at $a$. We will denote the Hessian matrix of $f$ at $a$ by $H(f)_{a}$. 


\subsection{On definable Morse functions.}

4.2.1. Critical points of functions. Let $M$ be a definable $C^{r}$-manifold of dimension $n$, with $r \geqslant 2$, and $f: M \rightarrow \mathbf{R}$ a definable $C^{2}$-function.

As usual, we say that a point $p \in M$ is a critical point of $f$ if the differential of $f$ at $p$ is zero. If $p$ is a critical point of $f$, then $f(p)$ is called a critical value of $f$.

Let $p$ be a critical point of $f$ and $\langle U, u\rangle$ a definable $C^{2}$-coordinate system on $M$ at $p$ (i.e., $U$ is an open definable subset of $M$ containing $p$ and $u$ is a $C^{2}$ diffeomorphism from $U$ onto an open subset of $\mathbf{R}^{n}$ with $\left.u(p)=0\right)$. The critical point $p$ is called nondegenerate if the Hessian matrix of $f \circ u^{-1}$ at 0 is nonsingular. If $v: V \rightarrow \mathbf{R}^{n}$ is another definable $C^{2}$-coordinate system on $M$ at $p$, then, by direct computations, the Hessian matrix of $f \circ v^{-1}$ at 0 is

$$
H\left(f \circ v^{-1}\right)_{0}=\left(J\left(v \circ u^{-1}\right)_{0}\right)^{T} H\left(f \circ u^{-1}\right)_{0} J\left(v \circ u^{-1}\right)_{0} .
$$

Hence, the notion of nondegeniricity does not depend on the choice of a local coordinate system.

If $p$ is a nondegenerate critical point of $f$ and $u: U \rightarrow \mathbf{R}^{n}$ a definable $C^{2}$ coordinate system on $M$ at $p$, then the Hessian $H\left(f \circ u^{-1}\right)_{0}$ is a symmetric, hence diagonalizable, matrix. The index of $f$ at $p$ is defined to be the number of negative eigenvalues of $H\left(f \circ u^{-1}\right)_{0}$ (counted with multiplicity). It follows from (4.1) that this number does not depend on the choice of a local coordinate system at $p$.

4.2.2. Definable Morse functions. Let $M$ be a definable $C^{2}$-manifold and $f: M \rightarrow$ $\mathbf{R}$ a definable $C^{2}$-function. As usual, we say that $f$ is a definable Morse function if it has finitely many critical points and all critical point of $f$ are nondegenerate. We say that $f$ has distinct critical values if $f\left(p_{1}\right) \neq f\left(p_{2}\right)$ for all critical points $p_{1} \neq p_{2}$.

For embedded definable manifolds the existence of definable Morse functions was proved in 9 .

Fact 4.1 (9, Theorem3]). Let $M$ be a definable $C^{r}$-submanifold of $\mathbf{R}^{m}, r \geqslant 2$. Then there is a definable $C^{r}$-function $f: \mathbf{R}^{m} \rightarrow \mathbf{R}$ such that the restriction of $f$ to $M$ is a definable Morse function on $M$ and all critical values of $f$ are distinct.

Since the proof of the following theorem is almost identical to the corresponding proof from [10, we will present it in Appendix A.

Theorem 4.2. Let $M$ be a definably compact $C^{4}$-manifold and $f: M \rightarrow \mathbf{R}$ a definable Morse $C^{4}$-function with distinct critical values. Let $p_{1}, \ldots, p_{n}$ be the critical points of $f$ and, for each $i=1, \ldots, n$, let $\lambda_{i}$ be the index of $f$ at $p_{i}$. Then the o-minimal Euler characteristic $\chi(M)$ of $M$ equals the sum $(-1)^{\lambda_{1}}+\cdots+(-1)^{\lambda_{n}}$.

4.3. Euler characteristic. Let $M$ be a definable $C^{p}$-submanifold of $\mathbf{R}^{m}$. The tangent bundle $T(M)$ of $M$ can be considered as a $C^{p-1}$-submanifold of the tangent bundle $T\left(\mathbf{R}^{m}\right)$ of $\mathbf{R}^{m}$. Since $T\left(\mathbf{R}^{m}\right)$ can be identified with $\mathbf{R}^{m} \times \mathbf{R}^{m}$, the tangent bundle $T(M)$ can be canonically identified with a $C^{p-1}$-submanifold of $\mathbf{R}^{m} \times \mathbf{R}^{m}$ (see [1, Section 2.5] for more details). The same procedure can be applied to the cotangent bundle $T^{*}(M)$ of $M$, and we may always consider both $T(M)$ and $T^{*}(M)$ as $C^{p-1}$-submanifolds of $\mathbf{R}^{m} \times \mathbf{R}^{m}$.

As usual, for a definable $C^{p}$-submanifold $M$ of $\mathbf{R}^{m}$ we say that the tangent bundle $T(M)$ is definably trivial if there is a definable $C^{p-1}$-isomorphism of vector 
bundles $\Theta: T(M) \rightarrow M \times \mathbf{R}^{n}$. Namely, $\Theta: T(M) \rightarrow M \times \mathbf{R}^{n}$ is a definable $C^{p-1}$ diffeomorphism such that for each $m \in M$, the restriction of $\Theta$ onto $T(M)_{m}$ is R-linear isomorphism from $T(M)_{m}$ onto $\{m\} \times \mathbf{R}^{n}$.

Theorem 4.3. Let $M$ be a definably compact $C^{4}$-submanifold of $R^{m}$ whose tangent bundle is definably trivial. Then the o-minimal Euler characteristic of $M$ is zero.

Proof. Considering definably connected components of $M$ separately, we are reduced to the case when $M$ is definably connected.

Let $\Theta: T(M) \rightarrow M \times \mathbf{R}^{n}$ be a definable $C^{3}$-isomorphism of vector bundles. For $x \in M$ we will use $\Theta$ to identify $T(M)_{x}$ with $\mathbf{R}^{n}$.

Let $U \subseteq M$ be an open subset, $h: U \rightarrow \mathbf{R}^{m}$ a definable $C^{1}$-map, and $x \in U$. The differential $\mathrm{D}(h)_{x}$ of $h$ at $x$ is a linear map from $T(M)_{x}$ to $\mathbf{R}^{m}$. Using $\Theta$ we identify $T(M)_{x}$ with $\mathbf{R}^{n}$, and consider $\mathrm{D}(h)_{x}$ as a linear map from $\mathbf{R}^{n}$ to $\mathbf{R}^{m}$. We will denote by $\left[D(h)_{x}\right]$ the corresponding $m \times n$-matrix. If $V \subseteq \mathbf{R}^{l}$ is a definable open set, $g: V \rightarrow M$ a definable $C^{1}$-map, and $y \in V$, then identifying again $T(M)_{f(y)}$ with $\mathbf{R}^{n}$, in the same way as above, we can consider $\mathrm{D}(g)_{y}$ as a linear map from $\mathbf{R}^{l}$ to $\mathbf{R}^{n}$ and will denote by $\left[D(g)_{y}\right]$ the corresponding matrix. If $h$ and $g$ are as above and in addition $x=h(y)$, then, by the Chain Rule, we have

$$
\left[D(h)_{x}\right]\left[D(g)_{y}\right]=J(h \circ g)_{y} .
$$

Claim 4.4. $M$ is definably orientable.

Proof of Claim. Let $\left\{\left\langle U_{i}, \varphi_{i}\right\rangle: I\right\}$ be a definable $C^{4}$-atlas such that every $U_{i}$ is definably connected. The map $x \mapsto \operatorname{det}\left[D\left(\varphi_{i}\right)_{x}\right]$ has constant sign on each $U_{i}$. We can apply Lemma 3.8 with $\lambda(i)$ being the sign.

We fix a definable orientation $\mathcal{A}$ on $M$, and changing it to the opposite if needed, we may assume that for all $\langle U, \varphi\rangle \in \mathcal{A}$ and $x \in U$ the determinant of $\left[D(\varphi)_{x}\right]$ is positive.

By Fact 4.1 we can choose a definable Morse $C^{4}$-function $f: M \rightarrow \mathbf{R}$ with distinct critical values. Let $p_{1}, \ldots, p_{k}$ be all critical points of $f$ and $\lambda_{i}, i=1, \ldots, k$, the correspondent indexes.

If $x \in M$, then $\left[\mathrm{D}(f)_{x}\right]$ is an $n \times 1$-matrix, and its transpose is a vector in $\mathbf{R}^{n}$. We will denote this vector by $\Omega(x)$. Thus we obtain a map $\Omega: M \rightarrow \mathbf{R}^{n}$ and it is not hard to see that it is a definable $C^{3}$-map. Since $M$ is definably compact, by Claim $[3.18, \operatorname{deg}(\Omega)=0$. Our goal is to show that this degree equals the Euler characteristic of $M$.

Obviously, $\Omega(x)=0$ if and only if $x$ is a critical point of $f$, hence $\Omega^{-1}(0)=$ $\left\{p_{1}, \ldots, p_{k}\right\}$. If 0 is a regular value of $\Omega$, then $0=\operatorname{deg}(\Omega)=\operatorname{deg}^{0}(\Omega)$. Hence, by Theorem 4.2, to finish the proof it is sufficient to show that each $p_{i}$ is a regular point of $\Omega$ and $\operatorname{deg}_{p_{i}}(\Omega)=(-1)^{\lambda_{i}}$.

We consider only the point $p_{1}$. We fix a chart $\langle U, \varphi\rangle \in \mathcal{A}$ with $p_{1} \in U$. Let $V=\varphi(U)$ and $q=\varphi\left(p_{1}\right)$. To show that $\operatorname{deg}_{p_{1}}(\Omega)=(-1)^{\lambda_{1}}$ it is sufficient to prove that the Jacobian of $\Omega \circ \varphi^{-1}$ at $q$ is a nonsingular matrix, and the sign of its determinant is $(-1)^{\lambda_{1}}$. According to the definition of the index of a function at a critical point, $(-1)^{\lambda_{1}}$ equals the sign of the determinant of the Hessian matrix $H\left(f \circ \varphi^{-1}\right)_{q}$, and this Hessian matrix is the Jacobian of $\nabla\left(f \circ \varphi^{-1}\right)$ at $q$. Thus to finish the proof we need to show that the sign of the determinant of $J\left(\Omega \circ \varphi^{-1}\right)_{q}$ is the same as the sign of the determinant of $J\left(\nabla\left(f \circ \varphi^{-1}\right)\right)_{q}$. 
According to the definition, for $x \in U$ and $y=\varphi(x)$, we have

$$
\Omega \circ \varphi^{-1}(y)=\left[\mathrm{D}(f)_{x}\right]^{T}
$$

and

$$
\nabla\left(f \circ \varphi^{-1}\right)(y)=\left(J\left(f \circ \varphi^{-1}\right)_{y}\right)^{T} .
$$

By (4.2),

$$
\left(J\left(f \circ \varphi^{-1}\right)_{y}\right)^{T}=\left(\left[\mathrm{D}(f)_{x}\right]\left[\mathrm{D}\left(\varphi^{-1}\right)_{y}\right]\right)^{T}=\left[\mathrm{D}\left(\varphi^{-1}\right)_{y}\right]^{T}\left[\mathrm{D}(f)_{x}\right]^{T} .
$$

Since $\left[\mathrm{D}\left(\varphi^{-1}\right)_{y}\right]=\left[\mathrm{D}(\varphi)_{x}\right]^{-1}$, we obtain

$$
\Omega \circ \varphi^{-1}(y)=B(y) \nabla\left(f \circ \varphi^{-1}\right)(y),
$$

where $B(y)$ denotes the transpose of the matrix $\left[D(\varphi)_{x}\right]$. Using standard differential calculus and taking into account that $q$ is a critical point of $f \circ \varphi^{-1}$, we obtain that the Jacobian of $\Omega \circ \varphi^{-1}$ at $q$ is equal to $B(q) J\left(\nabla\left(f \circ \varphi^{-1}\right)\right)_{q}$. Since the matrix $\left[D(\varphi)_{q}\right]=B(q)^{T}$ has positive determinant, the sign of the determinant of the Jacobian of $\Omega \circ \varphi^{-1}$ at $q$ is the same as the sign of the determinant of the Jacobian of $\nabla\left(f \circ \varphi^{-1}\right)$ at $q$.

Remark 4.5. We stated Theorem 4.3 only for affine definable manifolds. However, it can be applied also to abstract definable definably compact $C^{4}$-manifolds, since, by the Embedding Theorem [1], every such manifold is definably $C^{4}$-diffeomorphic to a definable $C^{4}$-submanifold of $\mathbf{R}^{m}$.

The following result was first proved by M. Edmundo.

Corollary 4.6. Let $G$ be a definably compact definable group. Then $\chi(G)=0$.

Proof. By [16, Proposition 3.1] (see also [12, Fact 1.10]), $G$ can be equipped with a structure of a definable $C^{4}$-manifold such that the group operation is also $C^{4}$. After applying the Embedding Theorem [1], we may assume that $G$ is a definable $C^{4}$ submanifold of $\mathbf{R}^{m}$. It is not hard to see that the tangent bundle of $G$ is definably trivial. Indeed, for $g \in G$ let $\alpha_{g}(x)$ be the diffeomorphism of $G$ defined as

$$
\alpha_{g}: x \mapsto g^{-1} x .
$$

The differential of $\alpha_{g}$ at $g$ maps $T(G)_{g}$ isomorphically onto $T(G)_{e}$. After fixing a basis for $T(G)_{e}$, we can identify definably $T(G)_{e}$ with $\mathbf{R}^{n}$, and use the map

$$
\Theta:(g, v) \mapsto\left(g, D\left(\alpha_{g}\right)_{g}(v)\right), \text { where } g \in G, v \in T(G)_{g},
$$

to obtain a trivialization of $T(G)$.

4.4. Lefschetz number. Let $X$ be a definably compact definably orientable definable $C^{p}$-manifold, with $p>2$. In [1] A. Berarducci and M. Otero introduced the Lefschetz number $\Xi(X)$ as the self-intersection number of the diagonal $\Delta_{X}$ in $X \times X$. They conjectured that, as in the classical case, $\Xi(X)$ coincides with the o-minimal Euler characteristic $\chi(X)$. In this section we prove this conjecture for the case $p=4$. 
Theorem 4.7. Let $X$ be a definably compact definably oriented definable $C^{4}$ manifold. Then $\Xi(X)=\chi(X)$.

Proof. Since the proof consists of putting together results of the previous section and results in [1, we will be brief.

We may assume that $X$ is $C^{4}$-submanifold of $\mathbf{R}^{m}$. We will denote by $n$ the dimension of $X$.

Let $X$ be as in the statement. By the Tubular Neighborhood Theorem 2 (see Appendix, Theorem A.4), there is an open neighborhood $U$ of $\Delta_{X}$ in $X \times X$ that is definably $C^{3}$-diffeomorphic to an open neighborhood of the zero section of the normal bundle of $\Delta_{X}$ in $X \times X$. It is not hard to see that the normal bundle of $\Delta_{X}$ in $X \times X$ is definably $C^{3}$-diffeomorphic to the cotangent bundle $T^{*}(X)$ of $X$. Therefore, we can find a definable $C^{3}$-diffeomorphism $\Phi$ from an open neighborhood $U$ of $\Delta_{X}$ in $X \times X$ onto an open neighborhood $V$ of the zero section of $X$ in $T^{*}(X)$.

We first use a Morse function to compute the self-intersection index of the zero section of $X$ in $T^{*}(X)$, and then apply $\Phi$ to compute $\Xi(X)$.

We consider $T^{*}(X)$ as a definable oriented $C^{3}$-manifold with the following orientation: Let $\langle U, \varphi\rangle$ be a definable $C^{4}$-chart on $X$ and $T(U)$ the subset $\bigcup_{a \in U}\{a\} \times$ $T(X)_{a}$ of $T(X)$. Then the pair $\left(T(U),\left(\varphi(x), \mathrm{D}(\varphi)_{x}\right)\right)$ is a definable $C^{3}$-chart on $T(X)$. If $\mathcal{A}$ is a definable $C^{4}$-atlas on $X$ such that $\langle X, \mathcal{A}\rangle$ is a definable oriented manifold, then $\left\{\langle(T(U),(\varphi, \mathrm{D}(\varphi))\rangle:(U, \varphi) \in \mathcal{A}\}\right.$ is a definable $C^{3}$-atlas on $T(X)$, and with this atlas $T(X)$ is a definable oriented manifold. Using the dot product on $\mathbf{R}^{m}$, we obtain an isomorphism between $T(X)$ and $T^{*}(X)$, and transfer this orientation to $T^{*}(X)$.

Let $f_{0}: \mathbf{R}^{m} \rightarrow \mathbf{R}$ be a definable $C^{4}$-function whose restriction $f=f_{0}\lceil X$ is a definable Morse function on $X$. The differential $D(f)$ of $f$ defines a $C^{3}$-section of the cotangent bundle $T^{*}(X)$. We will denote this section by $\delta$. Namely, $\delta$ is the $C^{3}$-map from $X$ into $T^{*}(X)$ that assigns to each $x$ in $X$ the differential of $f$ at $x$. Identifying $X$ with the zero section of $T^{*}(X)$, we obtain that $\delta(p) \in X$ if and only if $p$ is a critical point of $f$. Let $p$ be a critical point of $f$. Locally near $p$, the cotangent bundle $T^{*}(X)$ is trivializable. The same computations as in the previous section show that $\delta$ is transversal to $X$ at $p$, and the intersection number of $\delta$ and $X$ at $p$ is $(-1)^{\lambda}$, where $\lambda$ is the index of $f$ at $p$. By Theorem 4.2, the intersection number $I(\delta, X)$ is equal to the o-minimal Euler characteristic $\chi(X)$. It is left to show that $I(\delta, X)$ equals the self-intersection number of $\Delta_{X}$ in $X \times X$.

We first construct a definable homotopy from $\delta$ to the zero section of $T^{*}(X)$. Considering $T^{*}(X)$ as a definable submanifold of $\mathbf{R}^{m} \times \mathbf{R}^{m}$ we use the dot product from $\mathbf{R}^{m}$ to induce a dot product $\langle\cdot, \cdot\rangle$ and a norm $\|\cdot\|$ on each cotangent space $T^{*}(X)_{x}, x \in X$.

For $t \in[0,1]$ let $\delta_{t}: X \rightarrow T^{*}(X)$ be the section defined as $\delta_{t}(x)=(t\|\delta(x)\|) \delta(x)$. Since each $T^{*}(X)_{x}$ is an $\mathbf{R}$-vector space, these sections are well defined.

It is not hard to see that the map $\delta_{t}, t \in[0,1]$ is a definable proper $C^{3}$-homotopy between $\delta$ and the zero section. Moreover, for each $t \in(0,1]$ the section $\delta_{t}$ is transversal to the zero section and the intersection number $I\left(\delta_{t}, X\right)$ is the same as $I(\delta, X)$, namely $\chi(X)$.

Since $X$ is definably compact. we can find $\varepsilon>0$ such that $\delta_{t}(x) \in V$ for all $x \in X, t \in[0, \varepsilon)$. Since $\Phi$ is a $C^{3}$-diffeomorphism from $U$ to $V$, we can use $\Phi$ to transfer this information to $X \times X$ and obtain a definable $C^{3}$-submanifold $Y$ of $X \times X$ that is definably $C^{3}$-homotopic to $\Delta_{X}$ with $I\left(\Delta_{X}, Y\right)=\chi(X)$. 


\section{Appendix A. On Definable Morse functions}

We will outline the proof of Theorem 4.2. The arguments follow closely the corresponding proofs in [10, pages 4-21].

Let $M$ be a definably compact definable $C^{r}$-manifold and $f: M \rightarrow \mathbf{R}$ a definable $C^{r}$-function, where $r \geqslant 1$. Using the Embedding Theorem [1] we may assume that $M$ is a definable $C^{r}$-submanifold of $\mathbf{R}^{m}$. Also replacing $M$ with the graph of $f$ if needed, we may assume that $f$ is the projection onto the last coordinates.

For a definable subset $I \subseteq \mathbf{R}$ we denote by $M_{I}$ the set $f^{-1}(I)$, we use $M_{a}$ instead of $M_{\{a\}}$ for $I=\{a\}$.

Since $M$ is definably compact, $M_{\leqslant a}=\varnothing$ for all sufficiently large negative $a$, and $M_{\leqslant b}=M$ for all sufficiently large positive $b$. In particular, if $a$ is sufficiently large negative and $b$ sufficiently large positive, then $\chi(M)=\chi\left(M_{\leqslant b}\right)-\chi\left(M_{\leqslant a}\right)$. Thus, by the additivity of the Euler characteristic, Theorem 4.2 will follow from the following two statements.

Theorem A.1. Assume $r \geqslant 2$. Let $a<b \in \mathbf{R}$ be such that $[a, b]$ does not contain a critical value of $f$. Then $\chi\left(M_{\leqslant a}\right)=\chi\left(M_{\leqslant b}\right)$.

Theorem A.2. Assume $r \geqslant 4$. Let $q \in \mathbf{R}$ be a critical value of $f$ such that $f^{-1}(q)$ contains a unique critical point $p$, and $p$ is nondegenerate. Then for all sufficiently small $\varepsilon>0$,

$$
\chi\left(M_{\leqslant q+\varepsilon}\right)-\chi\left(M_{\leqslant q-\varepsilon}\right)=(-1)^{\lambda}
$$

where $\lambda$ is the index of $f$ at $p$

Before proving above theorems, we review tubular neighborhoods. We refer to [1] and [3] for more details.

A.1. Normal tubular neighborhoods. In this subsection we assume that $Z$ is a definable $C^{r}$-submanifold of $R^{m}$ of dimension $n$ with $r \geqslant 2$. We identify the tangent bundle $T(Z)$ with a subset of $\mathbf{R}^{m} \times \mathbf{R}^{m}$ in a natural way and consider it as a $C^{r-1}$-submanifold of $\mathbf{R}^{m} \times \mathbf{R}^{m}$.

Let $a \in Z$. The normal space of $Z$ at $a$, denoted by $\mathcal{N}(Z)_{a}$, is the set of all $v \in \mathbf{R}^{m}$ orthogonal to $T(Z)_{a}$. Obviously, each $\mathcal{N}(Z)_{a}$ is a vector subspace of $\mathbf{R}^{m}$ of dimension $m-n$. The normal bundle of $Z$ is

$$
\mathcal{N}(Z)=\bigcup_{a \in Z} a \times N(Z)_{a}
$$

It is a $C^{r-1}$-submanifold of $\mathbf{R}^{m} \times \mathbf{R}^{m}$ of dimension $m$.

For the proof of the following theorem we refer to [3, Theorem 6.11]. There it was proved for o-minimal structures over $\mathbb{R}$, but the same proof works for any o-minimal expansion of a field.

Theorem A.3 (Tubular Neighborhood Theorem 1). Let $Z$ be a definable $C^{r}$ submanifold of $\mathbf{R}^{m}$ with $r \geqslant 2$. Then there is an open neighborhood $U$ of the zero section $Z \times\{0\}$ in $\mathcal{N}(Z)$ such that the map $(a, v) \mapsto a+v$ is a $C^{r-1}$-diffeomorphism from $U$ onto an open neighborhood of $Z$ in $\mathbf{R}^{m}$.

Let $X$ be a definable closed $C^{r}$-submanifold of $Z$ of dimension $k$. For $a \in X$ the normal space of $X$ in $Z$ at $a$, that we will denote by $\mathcal{N}(X / Z)_{a}$, is the set $\left\{v \in T(Z)_{a}: v\right.$ is orthogonal to $\left.T(X)_{a}\right\}$. Obviously, $\mathcal{N}(X / Z)_{a}$ is a vector subspace of $T(Z)_{a}$ of dimension $n-k$. The normal bundle of $X$ in $Z$ is the set $\mathcal{N}(X / Z)=$ 
$\bigcup_{a \in X} \mathcal{N}(X / Z)_{a}$. It is easy to see that $\mathcal{N}(X / Z)$ is a definable $C^{r-1}$-submanifold of $T(Z)$ of dimension $n$. For $\varepsilon>0$ we will denote by $\mathcal{N}^{\varepsilon}(X / Z)$ the set $\{(a, v) \in$ $\mathcal{N}(X / Z):\|v\|<\varepsilon\}$.

Theorem A.4 (Tubular Neighborhood Theorem 2). Let $r \geqslant 2, Z$ a definable $C^{r}$-submanifold of $\mathbf{R}^{m}$ and $X$ a definably compact $C^{r}$-submanifold of $Z$. Then there is $\varepsilon>0$ and a definable $C^{r-1}$-diffeomorphism $\rho$ from $\mathcal{N}^{\varepsilon}(X / Z)$ onto an open neighborhood of $X$ in $Z$ such that $\rho(x, 0)=x$ for all $x \in X$.

Proof. Using Theorem A.3 we can find an open definable neighborhood $U$ of the zero section $Z \times\{0\}$ in $\mathcal{N}(Z)$ and an open definable neighborhood $V$ of $Z$ in $\mathbf{R}^{m}$ such that the map $(a, v) \mapsto a+v$ is a $C^{r-1}$-diffeomorphism from $U$ onto $V$.

Let $\pi: V \rightarrow Z$ be the map that assigns to $v \in V$ the unique $a \in Z$ such that $v=a+w$ for some $(a, w) \in U$. Obviously, $\pi$ is a definable $C^{r-1}$-map. Since $X$ is definably compact, there is $C>0$ such that $x+w \in U$ for all $x \in X$ and $w \in \mathbf{R}^{m}$ with $\|w\|<C$.

Consider the map $\rho: \mathcal{N}^{C}(X / Z) \rightarrow Z$ defined as $(x, w) \mapsto \pi(x+w)$. It is not hard to see that $\rho$ is a definable $C^{r-1}$-map with $\rho(x, 0)=x$, and the differential of $\rho$ at $(x, 0)$ is invertible for all $x \in X$. Using definable compactness of $X$ we can find $\varepsilon>0$ such that the differential of $\rho$ is invertible at all $(x, w) \in \mathcal{N}^{\varepsilon}(X / M)$.

A.2. Proof of Theorem A.1, Theorem A.1 will follow from the following theorem.

Theorem A.5. Let $M$ be a definably compact $C^{2}$-submanifold of $\mathbf{R}^{m}$ and $f: M \rightarrow$ $\mathbf{R}$ a definable $C^{2}$-function. If $a \in \mathbf{R}$ is a regular value of $f$, then there is an open interval I containing a such that $f$ has a definable trivialization over $I$.

Proof. We need to find a definable $X \subseteq \mathbf{R}^{m}$ and a definable homeomorphism $\varphi: X \times I \rightarrow M_{I}$ such that the following diagram is commutative:

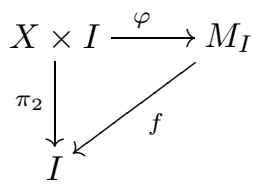

Considering the graph of $f$ if needed, we may assume that $f$ is the projection onto the last coordinate.

Let $X=f^{-1}(a)$. Since $a$ is a regular value of $f$, by Lemma 3.1 in [1, $X$ is a closed $C^{1}$-submanifold of $M$. In particular, $X$ is definably compact.

Denoting by $\xi \in \mathbf{R}^{m}$ the vector $(0, \ldots, 0,1)$, we obtain that $f(x)=\langle\xi, x\rangle$. Since $X$ is a submanifold of $M$ of co-dimension one, for every $x \in X$ the vector space $\mathcal{N}(X / M)_{x}$ is one-dimensional and, since $a$ is a regular value of $f$, the vector $\xi$ is not orthogonal to it. Thus for every $x \in X$ there is a unique vector $e_{x} \in \mathcal{N}(X / M)_{x}$ such that $\left\|e_{x}\right\|=1$ and $\left\langle\xi, e_{x}\right\rangle>0$. It is easy to see that the map $x \mapsto e_{x}$ is a definable $C^{1}$-map on $X$.

Let $\varepsilon$ and $\rho$ be as in Theorem A.4 and $J=(-\varepsilon, \varepsilon)$. Consider the function $\psi: X \times J \rightarrow \mathbf{R}$ defined as $(x, t) \mapsto f\left(\rho\left(x, t e_{x}\right)\right)$. It is not hard to see that for each $x \in X$ the differential of the function $t \mapsto \psi(x, t)$ at 0 is not zero.

Since $\psi(x, 0)=a$ for $x \in X$, by the Implicit Function Theorem, for every $x \in X$ there is an open $U_{x} \subseteq X \times \mathbf{R}$ containing $(x, a)$ and a definable $C^{1}$-function $\beta_{x}: U_{x} \rightarrow J$ such that $\psi\left(y, \beta_{x}(y, \tau)\right)=\tau$ for all $(y, \tau) \in U_{x}$. Using definable 
compactness of $X$ we can find an open interval $I \subseteq \mathbf{R}$ containing $a$ and a definable function $\beta: X \times I \rightarrow J$ such that $\psi(y, \beta(y, \tau))=\tau$ for all $(y, \tau) \in X \times I$.

It is easy to see that the map $\varphi:(y, \tau) \mapsto \rho(\tau, \beta(y, \tau))$ is a trivialization of $f$ over $I$.

Now we are ready to prove Theorem A.1 Let $M$ and $a<b$ be as in Theorem A.1. We can find an open interval $I$ containing $a, b$ such that $I$ does not contain a critical value of $f$. By o-minimality, the function $t \mapsto \chi\left(M_{t}\right)$ is definable. By Theorem A.5, it is locally constant on $I$, hence it has a constant value $C$ on $I$. Then

$$
\chi\left(M_{\leqslant b}\right)-\chi\left(M_{\leqslant a}\right)=\chi\left(M_{(a, b]}\right)=C \chi((a, b])=0 .
$$

Theorem A.1 is proved.

A.3. Proof of Theorem A.2. The following lemma and its proof are due to Chris Miller.

Lemma A.6 (C.Miller). Let $U$ be an open subset of $\mathbf{R}^{n}$ containing $0, r \geqslant 1$, and $f: U \rightarrow R$ a definable $C^{r}$-function with $f(0)=0$. Then there are definable $C^{r-1}$-functions $f_{i}: U \rightarrow \mathbf{R}$ such that $f=x_{1} f_{1}(x)+\cdots+x_{n} f_{n}(x)$ on $U$.

Proof. By induction on $n$.

The case $n=1$ : Let

$$
f_{1}(x)= \begin{cases}f(x) / x & x \neq 0, \\ f^{\prime}(0) & x=0 .\end{cases}
$$

It is easy to see that $f_{1}$ is $C^{r-1}$ and $f(x)=x f_{1}(x)$.

The case $n+1$ : By induction hypothesis we can find definable $C^{r-1}$-functions $f_{1}, \ldots, f_{n}$ such that $f\left(x_{1}, \ldots, x_{n}, 0\right)=x_{1} f_{1}\left(x_{1}, \ldots, x_{n}\right)+\cdots+x_{n} f_{n}\left(x_{1}, \ldots, x_{n}\right)$. We define $f_{n+1}\left(x_{1}, \ldots, x_{n+1}\right)$ as

$$
f_{n+1}\left(x_{1}, \ldots, x_{n+1}\right)= \begin{cases}f\left(x_{1}, \ldots, x_{n+1}\right) / x_{n+1} & \text { if } x_{n+1} \neq 0 \\ \frac{\partial f}{\partial x_{n+1}}\left(x_{1}, \ldots, x_{n}, 0\right) & \text { if } x_{n+1}=0 .\end{cases}
$$

It is easy to check that the functions $f_{i}$ satisfy the requirement.

Lemma A.7 (Morse's Lemma). Let $r \geqslant 0, M$ a definable $C^{r+2}$-manifold of dimension $n, f: M \rightarrow \mathbf{R}$ a definable $C^{r+2}$-function and $p \in M$ a nondegenerate critical point of $f$. Then there is a definable $C^{r}$-coordinate system $\langle U, \varphi\rangle$ on $M$ at $p$ such that

$$
f \circ \varphi^{-1}(y)=f(p)-y_{1}^{2}-\cdots-y_{\lambda}^{2}+y_{\lambda+1}^{2}+y_{n}^{2},
$$

where $\lambda$ is the index of $f$ at $p$.

Proof. Using Lemma A.6 twice we can find a definable $C^{r+2}$-coordinate system $(W, \psi)$ on $M$ at $p$ such that

$$
f \circ \psi^{-1}(y)=f(p)+\sum_{i, j=1}^{n} b_{i j}(y) y_{i} y_{j}
$$

where $b_{i j}$ are definable $C^{r}$-functions. For the rest of the proof we refer to the proof of Lemma 6.1.1 in [7]. 
We now proceed with the proof of Theorem A.2.

Replacing $f$ with $f(x)-f(p)$ we may assume $f(p)=0$.

Let $\langle U, \varphi\rangle$ be as in Lemma A.7 with $r=4$. We have

$$
f(x)=-\varphi_{1}^{2}(x)-\cdots-\varphi_{\lambda}^{2}(x)+\varphi_{\lambda+1}^{2}(x)+\cdots+\varphi_{n}^{2}(x)
$$

on $U$.

Let $\varepsilon>0$ be sufficiently small such that the interval $[-\varepsilon,+\varepsilon]$ does not contain any other critical values of $f$ besides zero, and $\varphi(U)$ contains the open ball $B_{2 \varepsilon}$ of radius $2 \varepsilon$ centered at the origin.

We choose a definable $C^{2}$-function $\mu: \mathbf{R} \rightarrow \mathbf{R}^{\geqslant 0}$ such that

$$
\begin{gathered}
\mu(0)>\varepsilon, \\
\mu(t)=0 \text { for } t \geqslant 2 \varepsilon, \\
-1<\mu^{\prime}(t) \leqslant 0 \text { for all } t \in \mathbf{R} .
\end{gathered}
$$

It is not hard to see that such $\mu$ exists and we can even find a semi-algebraic one.

We consider a new function $g: M \rightarrow \mathbf{R}$ that coincides with $f$ outside of $U$ and

$$
g=f-\mu\left(\varphi_{1}^{2}+\cdots+\varphi_{\lambda}^{2}+2 \varphi_{\lambda+1}^{2}+\cdots+2 \varphi_{n}^{2}\right) \text { on } U .
$$

It is easy to see that $g$ is a definable $C^{2}$-function.

We introduce two new functions $\xi, \eta: U \rightarrow \mathbf{R}$ :

$$
\xi=y_{1}^{2}+\cdots+y_{\lambda}^{2} \text { and } \eta=y_{\lambda+1}^{2}+\cdots+y_{n}^{2} .
$$

In these notations we have

$$
f \circ \varphi^{-1}=-\xi+\eta \text { and } g \circ \varphi^{-1}=-\xi+\eta-\mu(\xi+2 \eta) .
$$

Claim A.8. The critical points of $g$ and $f$ are the same.

Proof. Since $f$ and $g$ coincide outside of $U$, we need to consider only points in $U$. Working in the chart $\langle U, \varphi\rangle$, computing partial derivatives, and taking into account that $-1<\mu^{\prime} \leqslant 0$, it is easy to see that all partial derivatives of $g \circ \varphi^{-1}$ vanish simultaneously only at 0 .

Claim A.9. $g^{-1}((-\infty, \varepsilon])=f^{-1}((-\infty, \varepsilon])$.

Proof. Since $g(x) \leqslant f(x)$ everywhere on $M$, we have $f^{-1}((-\infty, \varepsilon]) \subseteq g^{-1}((-\infty, \varepsilon])$. Thus we only need to show the opposite inclusion.

Let $m \in g^{-1}((-\infty, \varepsilon])$.

If $m \notin U$, then $f(m)=g(m)$ and we are done.

Assume $m \in U$ and let $a=\varphi(m)$. If $\xi(a)+2 \eta(a) \geqslant 2 \varepsilon$, then again $f(a)=g(a)$. Assume now that $\xi(a)+2 \eta(a)<2 \varepsilon$. It is not hard to see that the maximum value of $-\xi+\eta$ on $\xi+2 \eta \leqslant 2 \varepsilon$ is $\varepsilon$. Hence $f(a)=-\xi(a)+\eta(a) \leqslant \varepsilon$ and $a \in$ $f^{-1}((-\infty,+\varepsilon])$.

From the previous claim we obtain

$$
\chi\left(M_{\leqslant+\varepsilon}\right)=\chi\left(g^{-1}((-\infty,+\varepsilon])\right) .
$$

Since $g(p)=-\mu(0)<-\varepsilon, g$ does not have critical values in $[-\varepsilon, \varepsilon]$. Hence, by Theorem A.1,

$$
\chi\left(g^{-1}((-\infty,+\varepsilon])\right)=\chi\left(g^{-1}((-\infty,-\varepsilon])\right) .
$$

To prove the theorem we need to show that

$$
\chi\left(g^{-1}((-\infty,-\varepsilon])\right)-\chi\left(M_{\leqslant-\varepsilon}\right)=(-1)^{\lambda} .
$$


Since $g \leqslant f$ on $M$, the set $M_{\leqslant-\varepsilon}$ is contained in $g^{-1}((-\infty,-\varepsilon])$, and the equation $($ 因) is equivalent to

$$
\chi\left(g^{-1}((-\infty,-\varepsilon]) \backslash M_{\leqslant-\varepsilon}\right)=(-1)^{\lambda} .
$$

Since $f$ and $g$ coincide outside of $U$, and $\mu$ vanishes outside the set $\xi+2 \eta \geqslant 2 \varepsilon$, the theorem reduces to showing that for the sets

$$
\begin{aligned}
& S_{f}=\left\{y \in \mathbf{R}^{n}: \xi(y)+2 \eta(y)<2 \varepsilon,-\xi(y)+\eta(y) \leqslant-\varepsilon\right\} \text { and } \\
& S_{g}=\left\{y \in \mathbf{R}^{n}: \xi(y)+2 \eta(y)<2 \varepsilon,-\xi(y)+\eta(y)-\mu(\xi(y)+2 \eta(y)) \leqslant-\varepsilon\right\}
\end{aligned}
$$

we have

$$
\chi\left(S_{g} \backslash S_{f}\right)=(-1)^{\lambda} .
$$

The set $S_{g} \backslash S_{f}$ can be described by the following system of inequalities:

$$
\begin{aligned}
\xi+2 \eta & <2 \varepsilon, \\
-\xi+\eta-\mu(\xi+2 \eta) & \leqslant-\varepsilon, \\
-\xi+\eta & >-\varepsilon .
\end{aligned}
$$

The set $\left\{(\eta, \xi) \in \mathbf{R}^{2}: \xi, \eta \geqslant 0\right\}$ satisfying

the above inequalities consists of 4 com-

ponents:

The origin $(0,0)$;

a point $(P, 0)$;

an open interval $\{(t, 0): 0<t<P\}$;

an open interval $\{(0, t): 0<t<\varepsilon\}$;

an open 1-cell $L$ in

$\mathbf{R}^{>0} \times \mathbf{R}^{>0}$

and an open 2-cell $D \subseteq \mathbf{R}^{>0} \times \mathbf{R}^{>0}$.

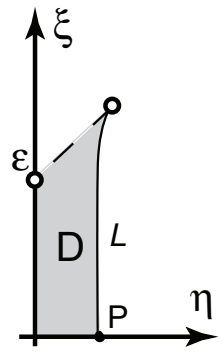

For $t>0$ the set $\xi^{-1}(t)$ is $(\lambda-1)$-sphere and its o-minimal Euler characteristic is $(-1)^{\lambda-1}+1$. The o-minimal Euler characteristic of a half-open interval is 0 , and also $\chi(D \cup L)=0$. Hence

$$
\chi\left(S_{g} \backslash S_{f}\right)=1-\left[(-1)^{\lambda-1}+1\right]=(-1)^{\lambda} .
$$

This finishes the proof of Theorem A.2.

\section{REFERENCES}

[1] Berarducci, Alessandro, and Otero, Margarita, Intersection theory for o-minimal manifolds, Ann. Pure Appl. Logic, 107, (2001), no. 1-3, 87-119. MR1807841 (2001m:03074)

[2] Berarducci, Alessandro, and Otero, Margarita, Transfer methods for o-minimal topology, J. Symbolic Logic, 68, (2003), no. 3, 785-794. MR2000077 (2004h:03082)

[3] Coste, Michel, An introduction to O-minimal geometry, Raag Publications.

[4] van den Dries, Lou, Tame topology and o-minimal structures, London Mathematical Society Lecture Note Series, 248, Cambridge University Press, Cambridge, 1998. MR1633348 (99j:03001)

[5] Dubrovin, B. A., Fomenko, A. T., and Novikov, S. P., Modern geometry-methods and applications. Part II, Graduate Texts in Mathematics, 104, The geometry and topology of manifolds; Translated from the Russian by Robert G. Burns, Springer-Verlag, New York, 1985. MR807945 (86m:53001)

[6] Edmundo, Mário J., Otero, Margarita, Definably compact abelian groups, J. Math. Log., 4, (2004), no. 2, 163-180. MR 2114966

[7] Hirsch, Morris W., Differential topology, Graduate Texts in Mathematics, 33, SpringerVerlag, New York, 1994. Corrected reprint of the 1976 original. MR1336822 (96c:57001) 
[8] Johns, Joseph, An open mapping theorem for o-minimal structures, J. Symbolic Logic, 66, (2001), no. 4, 1817-1820. MR.1877024 (2003f:03051)

[9] Ta Lé Loi, The existence of Morse functions on Sets definable in o-minimal structures, preprint.

[10] Milnor, J., Morse theory, Based on lecture notes by M. Spivak and R. Wells. Annals of Mathematics Studies, No. 51, Princeton University Press, Princeton, N.J., 1963. MR0163331 (29:634)

[11] Peterzil, Ya'acov, Some topological and differential invariants - a survey of the solution to the torsion-point problem, in Proceedings of the 2001 Ravello meeting in honor of A. Macintyre 60's birthday. MR 2159722 (2006d:03062)

[12] Peterzil, Y., Pillay, A., and Starchenko, S., Definably simple groups in o-minimal structures, Trans. Amer. Math. Soc., 352, (2000), no. 10, 4397-4419. MR.1707202 (2001b:03036)

[13] Peterzil, Ya'acov, and Starchenko, Sergei, Complex geometry and analytic-geometric categories, preprint (2005).

[14] Peterzil, Y., Pillay, A., Starchenko, S., Definably simple groups in o-minimal structures, Trans. Amer. Math. Soc., 352, (2000), no, 10, 4397-4419. MR.1707202 (2001b:03036)

[15] Peterzil, Ya'acov, Steinhorn, Charles, Definable compactness and definable subgroups of o-minimal groups, J. London Math. Soc. (2), 59, (1999), no. 3, 769-786. MR1709079 (2000i:03055)

[16] Pillay, Anand, On groups and fields definable in o-minimal structures, J. Pure Appl. Algebra, 53, (1988), no. 3, 239-255. MR961362 (89i:03069)

[17] Razenj, Vladimir, One-dimensional groups over an o-minimal structure, Ann. Pure Appl. Logic, 53, (1991), no. 3, 269-277, MR1129780(93b:03053a)

[18] Shiota, Masahiro, Geometry of subanalytic and semialgebraic sets, Progress in Mathematics, 150, Birkhäuser Boston, Inc., Boston, MA, 1997. MR1463945 (99b:14061)

[19] Strzebonski, Adam W., Euler characteristic in semialgebraic and other o-minimal groups, J. Pure Appl. Algebra, 96, (1994), no. 2, 173-201. MR.1303545(95j:03067)

[20] Woerheide, A., O-minimal homology Ph.D. Thesis, University of Illinois at UrbanaChampaign, (1996).

[21] Wong, Kam-Chau, A fixed point theorem for o-minimal structures, MLQ Math. Log. Q., 49, (2003), no. 6, 598-602. MR2013720 (2004m:03143)

Department of Mathematics, University of Haifa, Haifa, Israel

E-mail address: kobi@math.haifa.ac.il

Department of Mathematics, University of Notre Dame, Notre Dame, Indiana 46556

E-mail address: starchenko.1@nd.edu 